\title{
Causes and consequences of variation in offspring body mass: meta-analyses in birds and mammals
}

\author{
Victor Ronget ${ }^{1 *}$, Jean-Michel Gaillard ${ }^{1}$, Tim Coulson ${ }^{2}$, Michael Garratt $^{3}$, \\ François Gueyffier ${ }^{1}$, Jean-Christophe Lega ${ }^{1}$ and Jean-François Lemaitre ${ }^{1}$ \\ ${ }^{1}$ Univ Lyon, Université Lyon 1; CNRS, UMR5558, Laboratoire de Biométrie et Biologie Evolutive, F-69622 Villeurbanne, France \\ ${ }^{2}$ Department of Zoology, University of Oxford, Oxford OX13PS, U.K. \\ ${ }^{3}$ Department of Pathology, University of Michigan Medical School, Ann Arbor, MI 48109, U.S.A.
}

\begin{abstract}
Early survival is highly variable and strongly influences observed population growth rates in most vertebrate populations. One of the major potential drivers of survival variation among juveniles is body mass. Heavy juveniles are better fed and have greater body reserves, and are thus assumed to survive better than light individuals. In spite of this, some studies have failed to detect an influence of body mass on offspring survival, questioning whether offspring body mass does indeed consistently influence juvenile survival, or whether this occurs in particular species/environments. Furthermore, the causes for variation in offspring mass are poorly understood, although maternal mass has often been reported to play a crucial role. To understand why offspring differ in body mass, and how this influences juvenile survival, we performed phylogenetically corrected meta-analyses of both the relationship between offspring body mass and offspring survival in birds and mammals and the relationship between maternal mass and offspring mass in mammals. We found strong support for an overall positive effect of offspring body mass on survival, with a more pronounced influence in mammals than in birds. An increase of one standard deviation of body mass increased the odds of offspring survival by $71 \%$ in mammals and by $44 \%$ in birds. A cost of being too fat in birds in terms of flight performance might explain why body mass is a less reliable predictor of offspring survival in birds. We then looked for moderators explaining the among-study differences reported in the intensity of this relationship. Surprisingly, sex did not influence the intensity of the offspring mass-survival relationship and phylogeny only accounted for a small proportion of observed variation in the intensity of that relationship. Among the potential factors that might affect the relationship between mass and survival in juveniles, only environmental conditions was influential in mammals. Offspring survival was most strongly influenced by body mass in captive populations and wild populations in the absence of predation. We also found support for the expected positive effect of maternal mass on offspring mass in mammals $\left(r_{\text {pearson }}=0.387\right)$. As body mass is a strong predictor of early survival, we expected heavier mothers to allocate more to their offspring, leading them to be heavier and so to have a higher survival. However, none of the potential factors we tested for variation in the maternal mass-offspring mass relationship had a detectable influence. Further studies should focus on linking these two relationships to determine whether a strong effect of offspring size on early survival is associated with a high correlation coefficient between maternal mass and offspring mass.
\end{abstract}

Key words: body size, individual heterogeneity, early survival, maternal size, maternal allocation.

* Address for correspondence (Tel: +33472 448142; E-mail: victor.ronget@univ-lyon1.fr) 


\section{GONTENTS}

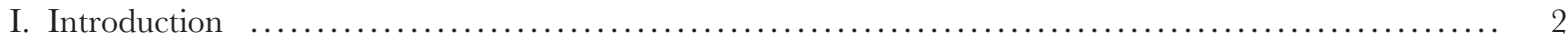

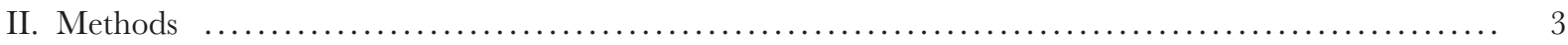

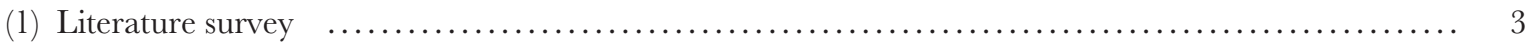

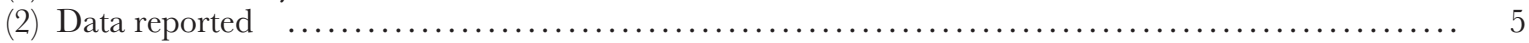

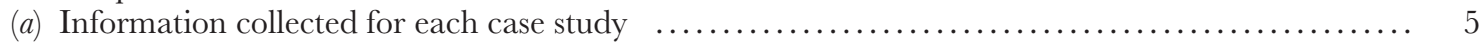

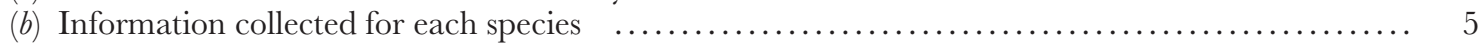

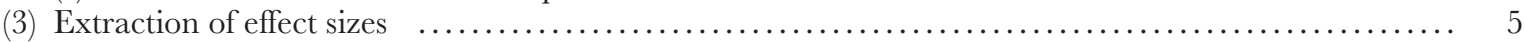

(a) Relationship between offspring mass and offspring survival $\ldots \ldots \ldots \ldots \ldots \ldots \ldots \ldots \ldots \ldots \ldots \ldots$

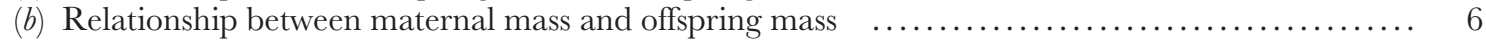

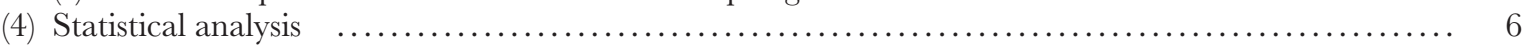

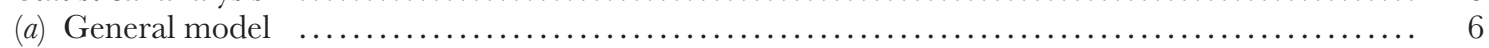

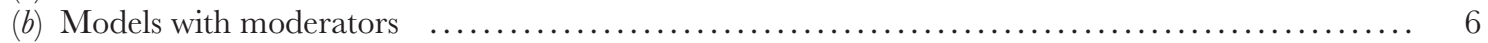

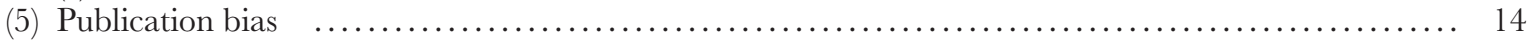

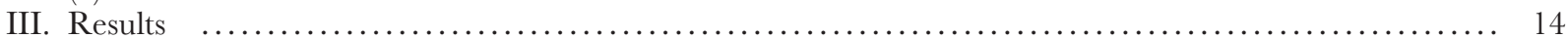

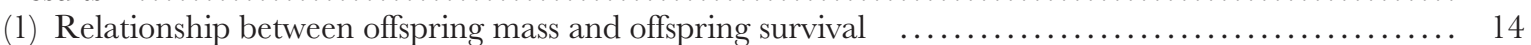

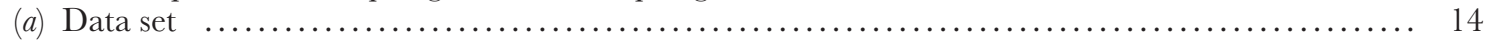

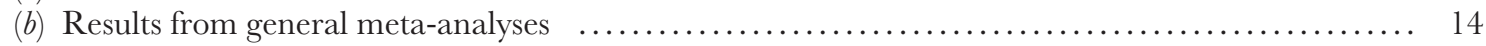

(c) Assessing the effects of moderators on the strength of the offspring mass-survival relationship $\quad \ldots \quad 16$

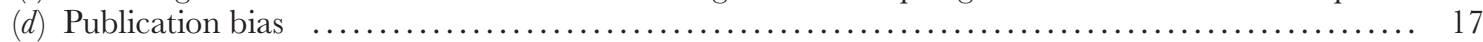

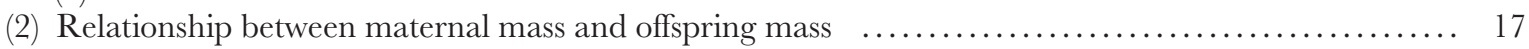

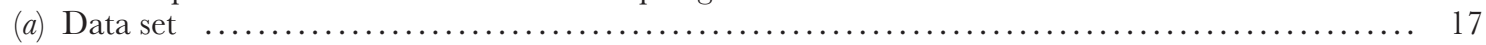

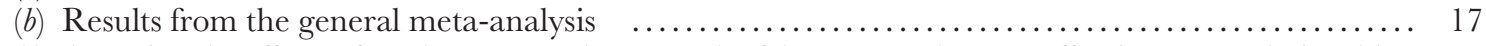

(c) Assessing the effects of moderators on the strength of the maternal mass-offspring mass relationship 17

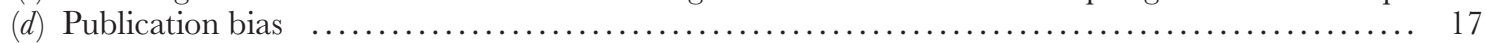

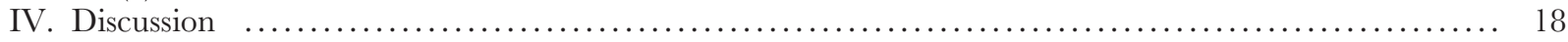

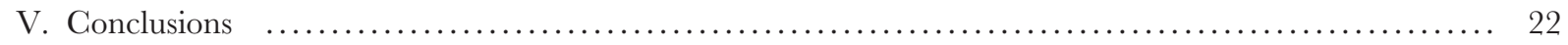

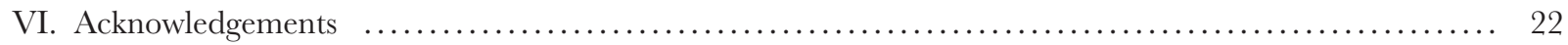

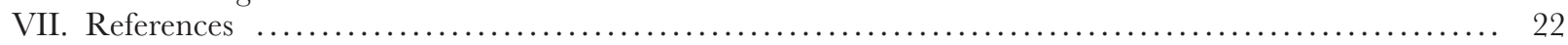

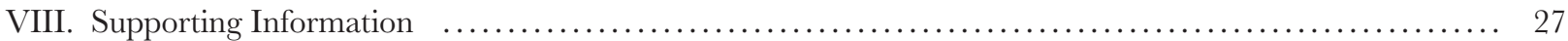

\section{INTRODUGTION}

Getting reliable estimates of demographic parameters including survival and reproduction is a major step in assessing population dynamics (Caswell, 2001). Individuals vary greatly in terms of lifespan and reproductive success, which lead them to differ strongly in their contribution to population dynamics. Life-history theory is built on the premise that individual traits that determine reproduction and survival throughout life are shaped by natural selection to maximize individual fitness (Gadgil \& Bossert, 1970; Stearns, 1992). It is therefore of crucial importance to assess the relationship among individual traits, demographic parameters, and individual fitness (Cam et al., 2002).

Juvenile survival is an important fitness component because it determines whether or not an individual will reach maturity and therefore reproduce (Lindström, 1999). In long-lived species of mammals and birds the juvenile period is a particularly critical life stage because mortality risks are much higher than after sexual maturity. Since offspring survival often drives population dynamics of long-lived species (Gaillard etal., 2000; Ozgul etal., 2010), it is particularly important to understand the ecological and biological factors that will modulate this fitness component. Numerous studies have investigated the influence of phenotypic traits on offspring survival, with a particular emphasis on body mass (Magrath, 1991; Maness \& Anderson, 2013). Generally, these studies have reported that body mass is a reliable predictor of offspring survival (e.g. Hamel et al., 2009; Mackas et al., 2010).

Body mass is known to be positively correlated with body fat, which represents the main component of body reserves in birds and mammals (Garnett, 1981; Labocha \& Hayes, 2012; Monteith etal., 2014; Bennett etal., 2015) and allows large individuals to survive over periods of food shortage. Furthermore, since body mass and body size are generally closely correlated across individuals within a given population, body size also has a positive effect on offspring survival (e.g. McMahon et al., 2015). For instance, in temperate ecosystems, individuals with greater body size survive better than those with low body reserves over the winter (Ringsby, Saether \& Solberg, 1998). In addition, energy demands for growth are high during the juvenile stage (Parker, Barboza \& Gillingham, 2009) and when food availability is low, body reserves allow growth to continue (Lee, Majluf \& Gordon, 1991). However, some studies have failed to detect a positive relationship between offspring body mass and juvenile survival (e.g. Williams \& Croxall, 1991; Ylönen, Horne \& Luukkonen, 2004; Reading etal., 2009). The most common explanation for these results involves quite constant and abundant food resources during the critical 
juvenile stage that lead body reserves, and consequently mass, to have less impact on survival (Van Vuren, Bray \& Heltzel, 2013). Likewise, in environments where most juvenile mortality is caused by predation, high individual body mass might not confer a particularly strong survival advantage (Warren, Mysterud \& Lynnebakken, 2001). Based on such contrasting results, it remains difficult to infer a general pattern for the effect of body mass on juvenile survival.

Among the factors that influence offspring body mass, maternal condition has been one of the most studied. Maternal body mass is indeed expected to account for a substantial proportion of the variation observed in offspring body mass (Pomeroy etal., 1999; Hamel, Craine \& Towne, $2012 a$ ) because heavy females can typically allocate more resources to their offspring during both pre- and post-natal stages (e.g. gestation and lactation in mammals), which leads to increased offspring mass and thereby offspring survival. Such relationships between maternal body mass and offspring body mass have been repeatedly documented in the literature (Clutton Brock etal., 1996; Monclús, Pang \& Blumstein, 2014). However, some case studies failed to detect such relationships (Campbell \& Slade, 1995; Wheatley et al., 2006; Foster \& Taggart, 2008). Common explanations for this inconsistency involve the offspring number-size trade-off (Michener, 1989), which appears to be the rule among short-lived species that produce multiple offspring per reproductive attempt (Smith \& Fretwell, 1974). Moreover, females of long-lived species often trade current allocation to reproduction for allocation to their own future survival (Tavecchia et al., 2005; Hamel etal., 2010). In harsh years, females of long-lived species are expected to put the emphasis on their own survival, which may produce costs in terms of losing their offspring or of producing offspring of reduced size (Skogland, 1984; Festa-Bianchet \& Jorgenson, 1998). Although Lim, Senior \& Nakagawa (2014) performed a pioneering meta-analysis to assess the direction of the relationship between mother and offspring body size and found support for an overall positive relationship, they included only a limited number of bird and mammal species (22 birds and 8 mammals) and did not identify the factors driving the observed variation in the strength of that relationship.

To fill this knowledge gap, we review empirical evidence of the strength of the relationships both between offspring body mass and offspring survival and between offspring mass and maternal body mass from published data. We restricted our analysis to birds and mammals because most detailed studies of free-ranging populations have been performed in these two vertebrate classes (Clutton-Brock \& Sheldon, 2010). We first performed two phylogenetically corrected meta-analyses (i.e. one for each relationship) to assess the direction and magnitude of these relationships. In a second step, we looked for biological factors that drive observed variation in each of the two relationships and could explain the conflicting results reported in literature.
We particularly focused on biological moderators that have previously been suggested to influence the relationships between mother and offspring mass, and juvenile survival. Offspring sex was included as one of these because male offspring of dimorphic and polygynous species are more susceptible to harsh conditions than females (Clutton-Brock, Albon \& Guinness, 1985). We thus expected that reserves and also body mass will have more influence on male than on female survival, which should ultimately lead to between-sex differences in the relationship between maternal mass and offspring mass. In addition, in polytocous species, the trade-off between offspring mass and offspring number should influence the relationship between offspring mass and maternal mass (Charnov \& Ernest, 2006). Thus we accounted for variation in litter size in the analysis of each relationship. Finally, we also tested for an influence of the species mating system because different mating systems lead to different patterns of maternal allocation (Zeveloff \& Boyce, 1980) and thereby to expected differences in the relationship between offspring mass and maternal mass.

\section{METHODS}

\section{(1) Literature survey}

We collected published papers by using the database of ISI Web of Science following a strict search protocol. The key words ('mass' or 'weight' or 'size') and ('survival' or 'mortality') were used to identify studies investigating relationships between offspring survival and mass and the key words ('mass' or 'weight') and ('mother' or 'maternal' or 'adult') and ('newborn' or 'offspring' or 'neonate') were used to identify studies testing for a relationship between maternal mass and offspring mass. The search was conducted in December 2015. We restricted the results to the topics 'Ecology', 'Zoology', 'Ornithology' and 'Evolutionary Biology'. We deliberately used broad key words because much of the required information can be hidden within papers on different topics (e.g. Serra etal., 2012). We identified 20240 papers related to offspring mass and survival and 1414 papers related to maternal mass and offspring mass. We applied a first selection procedure to this list based on the title and only retained papers dealing with mammalian or avian populations. Then, we read all the abstracts to check whether the relationships were explicitly reported in these papers. Finally, we checked the references cited in these articles for any relevant studies missed. A total of 103 papers on mammals and 133 papers on birds were retrieved for the relationship between offspring mass and survival (Fig. 1A). For the relationship between maternal mass and offspring mass we recovered 85 papers on mammals but only three papers on birds (Fig. 1B). We thus did not have enough data on birds to perform a meta-analysis for the relationship between maternal mass and offspring mass. This low amount of published data in birds is discussed in Section IV. 
(A)
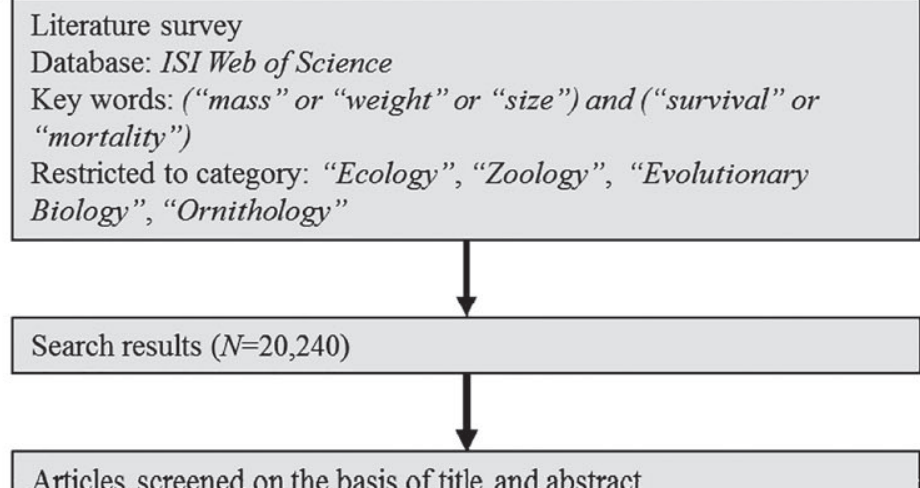

Articles screened on the basis of title and abstract
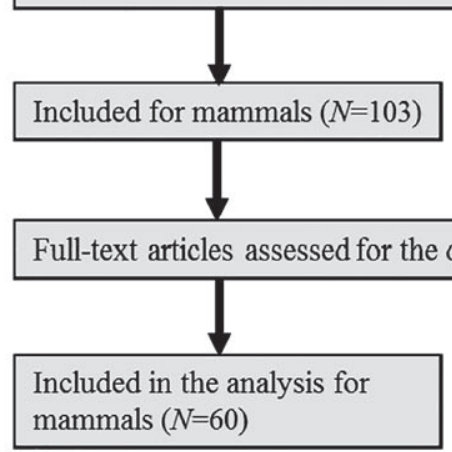

(B)
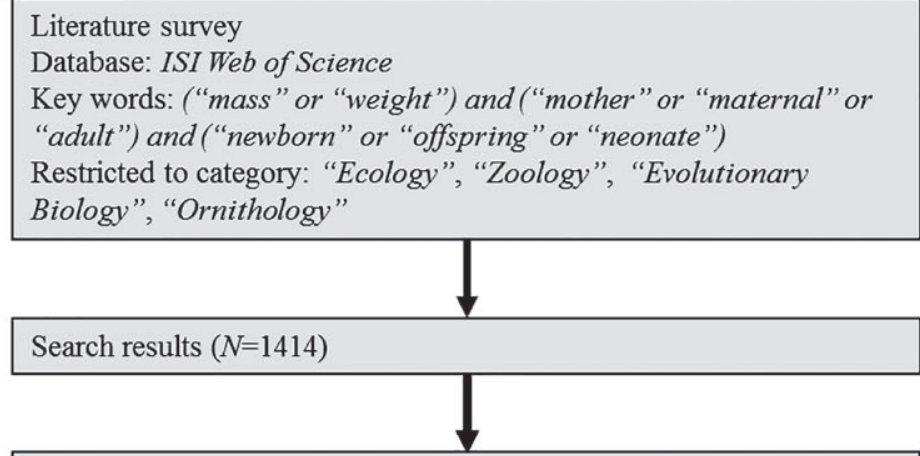

Articles screened on the basis of title and abstract

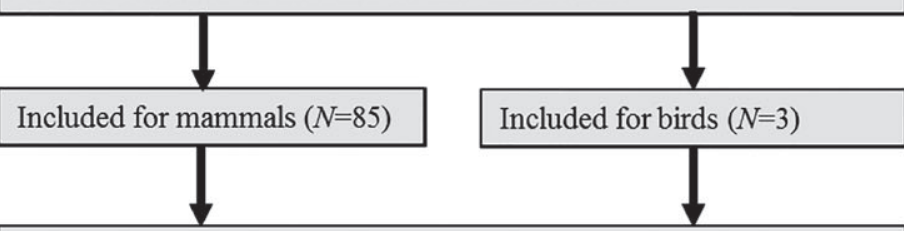

Full-text articles assessed for the quantitative analysis
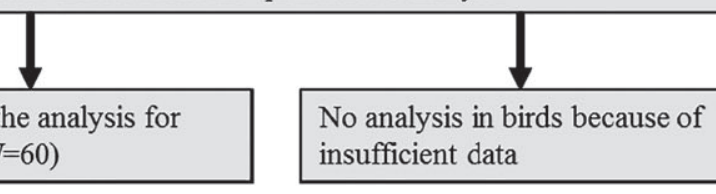

Fig. 1. PRISMA flow diagram [search procedure according to the PRISMA statement Liberati et al. (2009) and recommended by Nakagawa \& Poulin (2012)] for (A) the meta-analysis of the relationship between offspring mass and offspring survival and for (B) the meta-analysis of the relationship between maternal mass and offspring mass. 


\section{(2) Data reported}

\section{(a) Information collected for each case study}

For the relationship between offspring mass and survival and for the relationship between maternal mass and offspring mass we retained any relationship including mass or any indicator of mass such as structural size or body condition. When different measurements of mass were used in one paper, we extracted the strict measurement of mass. We did not consider pre-birth measurements such as egg or fetus mass. When the relationship was analysed at different ages (i.e. survival-offspring mass relationship at birth and at weaning), the earliest relationship was retained to avoid pseudo-replication due to repeated measures of the same individuals (Hurlbert, 1984). When the relationship was assessed independently for both sexes, we included sex-specific relationships in the analysis.

All information required for the identification of the paper (i.e. title, first author, year of publication, journal, location and species studied) was recorded. We also reported the timing of offspring measurement, the type of measurement and the data quality (see Section 2.4b), which could potentially influence the results of the meta-analysis. We included these factors as moderators in the meta-analysis. We also recorded whether the relationship was assessed for both sexes separately, or for pooled sexes. Lastly we reported whether the focal population was captive or not and if the individuals were subjected to predation.

\section{(b) Information collected for each species}

To assess the potential influence of biological factors on the relationships identified from our meta-analysis, we searched in the literature for information about mating system and litter size (mammal) or brood size (bird) for each species included in our data set (see online Appendices S1, S2 and S3 for all data used for the analysis).

\section{(3) Extraction of effect sizes}

\section{(a) Relationship between offspring mass and offspring survival}

This relationship was generally reported as a logistic function because survival follows a binomial distribution. The slope of the logistic regression was reported with its standard error. When the slope was not provided but the raw data or the logistic curve were graphically displayed in the paper, we extracted the data from the figure using WebPlotDigitizer (http://arohatgi.info/WebPlotDigitizer/) and then ran a logistic regression with the package betareg in $\mathrm{R}$ (version R.3.3.0, R Development Core Team 2015). In cases where the standard error was missing but the Wald statistics was reported, we used the Wald statistics to obtain the standard error. We calculated the Wald statistics as $\left(\theta-\theta_{0}\right)^{2} / \operatorname{var}(\theta)$, which is to be compared to a $\chi^{2}$ distribution with $\theta_{0}$ equal to 0 . When only the slope of the relationship was reported, the standard error could still be estimated when both the mean and the standard deviation of the offspring body mass were provided. We thus obtained the standard error by first simulating the survival data for each individual body mass using the published logistic relationship and then re-running a logistic regression (see $\mathrm{R}$ code in online Appendix S4). The relationship was sometimes presented with a quadratic term (e.g. Verboven \& Visser, 1998) and in such situations, when the raw data were available in the paper, we ran a new logistic regression without the quadratic term. This relationship was also sometimes presented as a linear relationship (e.g. Garnett, 1981). In such cases, we converted the slope of the linear regression to a logistic slope following the procedure given in Hamel, Yoccoz \& Gaillard (2012b). The linear relationship corresponds to a portion of a logistic that is quasi-linear, and multiplying the linear slope by a factor of 4 allows the slope of a logistic regression to be obtained. Occasionally, especially in old papers, the only results reported were the distributions of body mass with the mean and the standard deviation of the mass of dead and alive individuals. In such cases, we assumed that the masses of the dead individuals and of the live individuals were normally distributed and we simulated two normal distributions (one for each group) and ran a logistic regression. We replicated the procedure 10000 times and retained the mean slope and standard error of this slope (see $\mathrm{R}$ code in online Appendix S4).

When performing a meta-analysis, standardized coefficients are required to make results from the compiled studies comparable (Nakagawa \& Santos, 2012). Only the measurement of mass was standardized in our data set because the slopes were obtained from very different species that have markedly different distributions of offspring mass. We did not standardize survival because survival is bounded between and 0 and 1 across all case studies and species. To standardize body mass, the standard deviation of mass was required. When not available, the range of mass was used to infer the standard deviation. We assumed that mass was normally distributed, so that the range corresponds to 4 standard deviations (because in a normal distribution 95\% of the values belong to the interval encompassing approximatively two standard deviations). Semi-standard slopes were calculated by multiplying the slope with the standard error of the mass (Menard, 2011), the standard error being calculated in the same way. The effect sizes were reported in terms of odds ratios to facilitate interpretation (Lipsey \& Wilson, 2001). The odds ratio is calculated as the exponential of the semi-standardized slope. When mass increases by one standard deviation, the odds of survival (i.e. the ratio between the probability to survive and the probability to die) is multiplied by one semi-standardized odds ratio. Thus, a coefficient greater than 1 corresponds to a positive effect of mass on survival. To assess a potential impact of the data extraction on the results for each effect size we scored the data quality as high when all the required information was reported in the paper and as low when we needed to report the data from the figure or to run simulations to obtain the information (see Tables 1, 2 and 3 for detailed information on the extraction 
procedure of data and on the quality assessment of each paper).

\section{(b) Relationship between maternal mass and offspring mass}

For this relationship, the coefficients extracted were Pearson correlation coefficients or partial correlation coefficients. Correlation coefficients can also be inferred from $\chi^{2}, t$, and $F$ statistics using the formulae provided in Lipsey \& Wilson (2001). When only raw data were provided we extracted them with WebPlotDigitizer and ran the $\mathrm{R}$ function cor.test on the data. For the meta-analysis, all these correlation coefficients were converted into a Fisher $Z r$, which is an unbounded measure of effect size for correlation coefficients (Lipsey \& Wilson, 2001). This transformation allows the direct calculation of the standard error when the sample size is known. Following Cohen (1988), we considered that correlation coefficients of $0.1,0.3$, and 0.5 represent low, moderate, and strong effects, respectively. We reported the quality of the effect size as described in Section 2.3a to check whether our transformation had any impact on the results of the meta-analysis.

\section{(4) Statistical analysis}

\section{(a) General model}

A multi-level meta-analysis was performed because the effect sizes are not independent from each other. Correlation between the different effect sizes can arise when multiple effect sizes are recorded in one population or on the same species or in studies analysed by the same author. Moreover, the shared history among different species makes them non-independent (Harvey \& Pagel, 1991). For such analyses, linear mixed models are recommended (Nakagawa \& Santos, 2012). We used the function MCMCglmm of the package MCMCglmm (Hadfield, 2010; Hadfield \& Nakagawa, 2010) to perform our analyses. Bayesian hierarchical models are especially recommended to handle phylogenetic meta-analyses in which several effect sizes are reported for the same species (e.g. Santos \& Nakagawa, 2012).

In order to assess phylogenic relatedness among the different species, we used phylogenetic trees for avian (Jetz et al., 2012) and mammalian (Bininda-Emonds etal., 2007) species. These phylogenies were used in the meta-analyses to correct for non-independence between species-specific data points.

For each meta-analysis, linear mixed models were fitted with the effect size as the dependent variable and the error variance implemented for each effect size (with the mev argument in the function MCMCglmm). The covariance matrix among the species was extracted from the phylogeny. The phylogeny, species, population and first author were included in the model as random factors. We included another random effect as species independently of phylogeny because individuals from the same species can share characteristics that are independent of phylogeny (e.g. lifestyle). In the absence of clear a priori information, we used a non-informative prior (Inverse Wishart prior with $v=0.02$ and $V=1$ ). To assess whether the prior impacted the results, we re-ran the analysis using a new parameter expanded prior $(v=1, V=1$, alpha.mu $=0$, alpha. $V=1000)$. This sensitivity analysis did not uncover any difference between the two models, meaning that the results we obtained were not dependent on the prior used. Each model was run with 2000000 iterations. We ran several models and assessed convergence with the Gelmann diagnostic (Gelmann \& Rubin, 1992) by using the Gelmann.diag function in $\mathrm{R}$. This diagnostic detects statistically significant differences in the MCMC chains that could potentially occur between two models when these models do not converge. However, we did not detect any difference among models.

For each model, the mean of the posterior distribution was reported, which corresponds to the meta-analysis mean. We also reported the $95 \%$ credibility interval of the highest posterior density distribution (HPDI). The mean was considered as statistically significant when 0 (for $Z r$ ) or 1 (for the odds ratio) was not included in the credibility interval. To quantify the importance of the different random effects, $I^{2}$ statistics were calculated for each random effect (Nakagawa \& Santos, 2012). $I^{2}$ represents the percentage of the total variance that is accounted for by the random effect. Values of 25, 50, and $75 \%$ are classically interpreted as a low, moderate, and high percentage of variance explained, respectively (Higgins et al., 2003). The $I^{2}$ values are presented with their $95 \%$ highest posterior density credibility interval, this interval being bounded between 0 and 1 .

\section{(b) Models with moderators}

To test the effect of moderators we included them with fixed effects in new models. In addition to the biological variables presented in the introduction we included other study-specific variables that could influence the intensity of the relationships. We implemented the following moderators.

(1) The timing of the measurement, which is the life stage that includes the time elapsed between the offspring mass measurement and the record of offspring survivorship. The timing of the measurement was included in models as a three-level factor (Early, Late and Total). 'Early' corresponds to pre-weaning (mammals) or pre-fledging (birds) survival. Mass is then recorded at or right after birth (mammals) or hatching (birds). 'Late' corresponds to post-weaning (mammals) or post-fledging (birds) survival before recruitment. Mass is then recorded at or close to weaning (mammals) or fledging (birds). 'Total' corresponds to a survival estimate encompassing both pre- and post-weaning (mammals) or fledging (birds). Mass is then recorded at or right after birth (mammals) or hatching (birds). We also used the same kind of metrics for the relationship between offspring mass and maternal mass. We distinguished between pre- and 
Table 1. Summary of the statistics and of the potential driving factors compiled in the meta-analysis for the relationship between offspring mass and offspring survival in mammals. For each study the logistic slope (Beta), its standard error (S.E.) and the standard deviation of the mass distribution (S.D. Mass), the standardized logistic slope (standardized Beta) and the standardized standard error (standardized S.E.) are reported. The extraction procedure is reported in parentheses as 1 if the statistic is directly calculated in the study, 2 if data are reported from a figure, and 3 if data are reported from our own simulation. See Section II.3a for further information on the extraction procedure and Section II. $4 b$ for explanation of Timing of measurement categories

\begin{tabular}{|c|c|c|c|c|c|c|c|c|c|c|}
\hline Species & Study & Beta & S.E. & $\begin{array}{l}\text { S.D. } \\
\text { mass }\end{array}$ & $\begin{array}{l}\text { Standardized } \\
\text { beta }\end{array}$ & $\begin{array}{l}\text { Standardized } \\
\text { S.E. }\end{array}$ & $\begin{array}{l}\text { Mass or } \\
\text { condition }\end{array}$ & $\begin{array}{c}\text { Timing of } \\
\text { measurement }\end{array}$ & Sex & $\begin{array}{l}\text { Wild or } \\
\text { captive }\end{array}$ \\
\hline \multicolumn{11}{|l|}{ CARNIVORA } \\
\hline \multirow[t]{2}{*}{ Ursus maritimus } & $\begin{array}{l}\text { Ramsay \& Stirling } \\
\quad(1988)\end{array}$ & $0.012(3)$ & $0.007(3)$ & $32.1(3)$ & 0.385 & 0.225 & Mass & Early & Combined & Wild \\
\hline & $\begin{array}{l}\text { Derocher \& Stirling } \\
\quad(1996)\end{array}$ & $0.1423(1)$ & $0.054(3)$ & $3.02(3)$ & 0.430 & 0.163 & Mass & Early & Combined & Wild \\
\hline Ursus arctos & Dahle et al. (2006) & $0.089(1)$ & $0.055(1)$ & $7.517(1)$ & 0.669 & 0.413 & Mass & Late & Combined & Wild \\
\hline \multirow[t]{3}{*}{ Mirounga leonina } & $\begin{array}{l}\text { McMahon, Burton \& } \\
\text { Bester }(2000)\end{array}$ & $0.031(3)$ & $0.018(3)$ & $5.22(3)$ & 0.162 & 0.094 & Mass & Total & Female & Wild \\
\hline & & $0.04(3)$ & $0.015(3)$ & $6.02(3)$ & 0.241 & 0.090 & Mass & Total & Male & Wild \\
\hline & $\begin{array}{l}\text { Postma, Bester \& de } \\
\text { Bruyn (2013) }\end{array}$ & $0.024(1)$ & $0.019(1)$ & $20.62(1)$ & 0.495 & 0.392 & Mass & Late & Combined & Wild \\
\hline $\begin{array}{l}\text { Leptonychotes } \\
\text { weddellii }\end{array}$ & $\begin{array}{l}\text { Proffitt, Garrott \& } \\
\text { Rotella (2008) }\end{array}$ & $0.007(1)$ & $0.003(1)$ & $21.4(1)$ & 0.150 & 0.064 & Mass & Late & Combined & Wild \\
\hline \multirow[t]{2}{*}{ Phoca vitulina } & $\begin{array}{l}\text { Coltman, Bowen \& } \\
\text { Wright (1998) }\end{array}$ & $-0.21(3)$ & $0.36(3)$ & $1.02(3)$ & -0.214 & 0.367 & Mass & Early & Female & Wild \\
\hline & & $0.38(3)$ & $0.38(3)$ & $1.26(3)$ & 0.479 & 0.479 & Mass & Early & Male & Wild \\
\hline \multirow[t]{2}{*}{ Halichoerus grypus } & $\begin{array}{l}\text { Hall, McConnell \& } \\
\text { Barker (2001) }\end{array}$ & $0.353(1)$ & $0.159(1)$ & $1(1)$ & 0.353 & 0.159 & Condition & Late & Combined & Wild \\
\hline & $\begin{array}{l}\text { Hall, McConnell \& } \\
\text { Barker (2002) }\end{array}$ & $0.256(1)$ & $0.135(1)$ & $1(1)$ & 0.256 & 0.135 & Mass & Late & Combined & Wild \\
\hline \multirow[t]{2}{*}{ Eumetopias jubatus } & Maniscalco (2014) & $0.097(3)$ & $0.049(3)$ & $4.35(3)$ & 0.422 & 0.213 & Mass & Total & Male & Wild \\
\hline & & $0.096(3)$ & $0.063(3)$ & $3.75(3)$ & 0.360 & 0.236 & Mass & Total & Female & Wild \\
\hline $\begin{array}{l}\text { Zalophus } \\
\text { califormianus }\end{array}$ & Kraus et al. (2013) & $1.804(1)$ & $0.209(1)$ & $1.009(3)$ & 1.820 & 0.211 & Mass & Early & Combined & Wild \\
\hline Arctocephalus gazella & $\begin{array}{l}\text { Hoffman, Forcada \& } \\
\quad \text { Amos (2006) }\end{array}$ & $0.763(1)$ & $0.15(3)$ & $0.64(3)$ & 0.488 & 0.096 & Mass & Early & Combined & Wild \\
\hline \multicolumn{11}{|c|}{ ARTIODACTYLA } \\
\hline Vicugna vicugna & $\begin{array}{l}\text { Donadio, Buskirk \& } \\
\quad \text { Novaro }(2012)\end{array}$ & $0.773(1)$ & $0.269(1)$ & $0.957(1)$ & 0.740 & 0.257 & Mass & Early & Combined & Wild \\
\hline Lama guanicoe & Gustafson et al. (1998) & $0.067(3)$ & $0.114(3)$ & $2.18(3)$ & 0.146 & 0.249 & Mass & Early & Combined & Wild \\
\hline \multirow{2}{*}{$\begin{array}{l}\text { Antilocapra } \\
\text { americana }\end{array}$} & Fairbanks (1993) & $-0.01(3)$ & $0.99(3)$ & $0.45(3)$ & -0.005 & 0.446 & Mass & Early & Combined & Wild \\
\hline & $\begin{array}{l}\text { Van Vuren etal. } \\
\qquad(2013)\end{array}$ & $-0.91(3)$ & $0.61(3)$ & $0.47(3)$ & -0.428 & 0.287 & Mass & Early & Combined & Wild \\
\hline \multirow[t]{10}{*}{ Cerous elaphus } & $\begin{array}{l}\text { Blaxter \& Hamilton } \\
\quad(1980)\end{array}$ & $0.551(3)$ & $0.129(3)$ & $1.25(3)$ & 0.689 & 0.161 & Mass & Early & Combined & Captive \\
\hline & $\begin{array}{l}\text { Loison, Langvatn \& } \\
\quad \text { Solberg (1999) }\end{array}$ & $0.237(1)$ & $0.049(1)$ & $5(3)$ & 1.185 & 0.245 & Mass & Total & Combined & Wild \\
\hline & $\begin{array}{l}\text { Barber-Meyer, Mech } \\
\text { \& White (2008) }\end{array}$ & $-0.001(3)$ & $0.113(3)$ & $2.25(3)$ & -0.002 & 0.254 & Mass & Total & Female & Wild \\
\hline & & $0.001(3)$ & $0.128(3)$ & $2.64(3)$ & 0.003 & 0.338 & Mass & Total & Male & Wild \\
\hline & $\begin{array}{l}\text { White, Zager \& } \\
\text { Gratson }(2010)\end{array}$ & $0.101(1)$ & $0.033(1)$ & $3.71(3)$ & 0.375 & 0.122 & Mass & Early & Combined & Wild \\
\hline & & $0.043(1)$ & $0.031(1)$ & $3.97(3)$ & 0.171 & 0.123 & Mass & Early & Combined & Wild \\
\hline & Griffin et al. (2011) & $0.02(1)$ & $0.01(1)$ & $2.6(3)$ & 0.052 & 0.026 & Mass & Early & Combined & Wild \\
\hline & Moyes et al. (2011) & $0.55(1)$ & $0.06(1)$ & $0.375(3)$ & 0.206 & 0.023 & Mass & Total & Combined & Wild \\
\hline & Walling et al. (2011) & $0.58(1)$ & $0.057(1)$ & $2(3)$ & 1.160 & 0.114 & Mass & Total & Combined & Wild \\
\hline & Stopher et al. (2014) & $0.23(1)$ & $0.02(1)$ & $1(1)$ & 0.230 & 0.020 & Mass & Total & Combined & Wild \\
\hline \multirow{3}{*}{$\begin{array}{l}\text { Odocoileus } \\
\quad \text { virginianus }\end{array}$} & Sams etal. (1996) & $0.596(1)$ & $0.40(3)$ & $0.972(3)$ & 0.579 & 0.389 & Mass & Early & Combined & Wild \\
\hline & Ditchkoff et al. (2001) & $0.533(1)$ & $0.335(1)$ & $2.57(3)$ & 1.370 & 0.861 & Condition & Early & Combined & Wild \\
\hline & Carstensen et al. (2009) & $1.50(3)$ & $0.63(3)$ & $0.77(3)$ & 1.155 & 0.485 & Mass & Early & Combined & Wild \\
\hline \multirow[t]{5}{*}{ Odocoileus hemionus } & White et al. (1987) & $0.113(3)$ & $0.033(3)$ & $4.22(3)$ & 0.477 & 0.139 & Mass & Late & Combined & Wild \\
\hline & $\begin{array}{l}\text { Bishop, Unsworth \& } \\
\text { Garton (2005) }\end{array}$ & $0.195(1)$ & $0.072(1)$ & $4.74(3)$ & 0.924 & 0.341 & Mass & Late & Combined & Wild \\
\hline & $\begin{array}{l}\text { Lomas \& Bender } \\
\quad(2007)\end{array}$ & $0.19(1)$ & $0.04(1)$ & $0.817(3)$ & 0.155 & 0.033 & Mass & Early & Combined & Wild \\
\hline & Bishop et al. (2009) & $0.446(3)$ & $0.145(3)$ & $0.9(3)$ & 0.401 & 0.131 & Mass & Early & Combined & Wild \\
\hline & Hurley et al. (2011) & $0.194(1)$ & $0.113(1)$ & $1.5(3)$ & 0.291 & 0.170 & Mass & Early & Combined & Wild \\
\hline \multirow[t]{2}{*}{ Rangifer tarandus } & Whitten et al. (1992) & $-0.31(3)$ & $0.37(3)$ & $0.989(3)$ & -0.307 & 0.366 & Mass & Early & Female & Wild \\
\hline & & $0.52(3)$ & $0.29(3)$ & $1.247(3)$ & 0.648 & 0.362 & Mass & Early & Male & Wild \\
\hline
\end{tabular}


Table 1. Continued

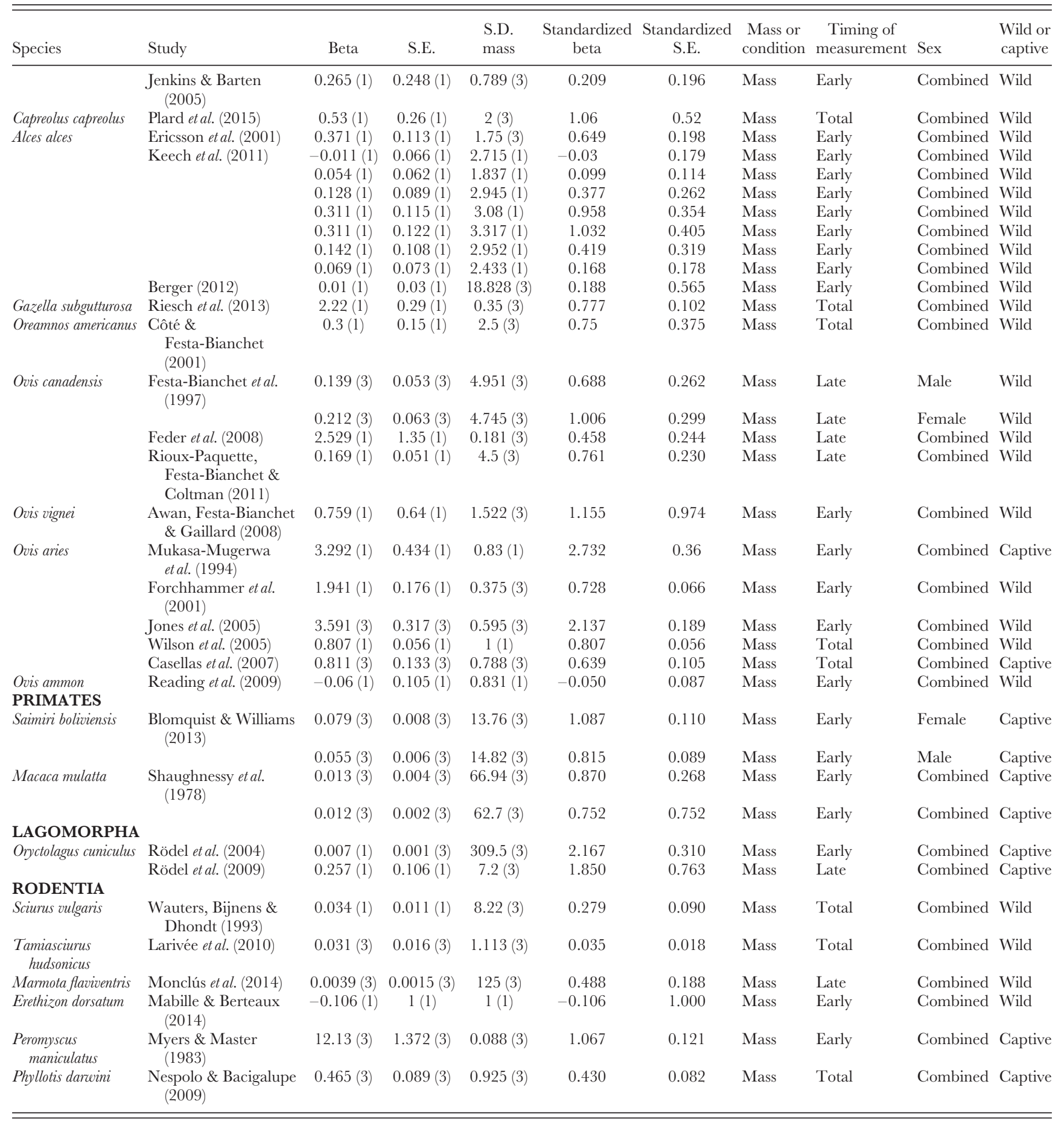

post-weaning (mammals) or fledging (birds) periods when possible because the weaning (mammals) and fledging (birds) periods are usually the most critical life stages (Clutton-Brock, 1991). In particular, at weaning, most mammals no longer rely on parental care for survival.

(2) The type of mass measurement was fitted as a two-level factor (Mass versus Condition index). We included this moderator to assess whether the use of different measures impacted our results. In some cases, condition index and mass can be related to body reserves with different intensities (e.g. Wilder, Raubenheimer \& Simpson, 2016).

(3) The fact that the data were obtained from wild or captive conditions was recorded as a two-level factor (Wild versus Captive). We considered a population as 
Table 2. Summary of the statistics and potential driving factors compiled in the meta-analysis for the relationship between offspring mass and offspring survival in birds. For each study the logistic slope (Beta), its standard error (S.E.) and the standard deviation of the mass distribution (S.D. Mass), the standardized logistic slope (standardized Beta) and the standardized standard error (standardized S.E.) are reported. The extraction procedure is reported in parentheses as 1 if the statistic is directly calculated in the study, 2 if data are reported from a figure, and 3 if data are reported from our own simulation. See Section II.3 $a$ for further information on the extraction procedure and Section II. $4 b$ for explanation of Timing of measurement categories

\begin{tabular}{|c|c|c|c|c|c|c|c|c|c|c|}
\hline Species & Study & Beta & S.E. & $\begin{array}{l}\text { S.D. } \\
\text { mass }\end{array}$ & $\begin{array}{l}\text { Standardized } \\
\text { beta }\end{array}$ & $\begin{array}{l}\text { Standardized } \\
\text { S.E. }\end{array}$ & $\begin{array}{l}\text { Mass or } \\
\text { condition }\end{array}$ & $\begin{array}{l}\text { Timing of } \\
\text { measurement }\end{array}$ & Sex & $\begin{array}{l}\text { Wild or } \\
\text { captive }\end{array}$ \\
\hline \multicolumn{11}{|l|}{ CORACIIFORMES } \\
\hline \multicolumn{11}{|c|}{ STRIGIFORMES } \\
\hline \multirow[t]{2}{*}{ Athene cunicularia } & Todd et al. (2003) & $0.053(3)$ & $0.032(2)$ & $14.24(3)$ & 0.755 & 0.456 & Mass & Late & Combined & Wild \\
\hline & $\begin{array}{l}\text { Davies \& Restani } \\
\quad(2006)\end{array}$ & $-0.032(3)$ & $0.35(2)$ & $0.99(3)$ & -0.032 & 0.347 & Condition & Total & Combined & Wild \\
\hline \multicolumn{11}{|c|}{ FALGONIFORMES } \\
\hline Accipiter gentilis & $\begin{array}{l}\text { Wiens, Noon \& } \\
\text { Reynolds (2006) }\end{array}$ & $0.01(1)$ & $0.01(1)$ & $110(3)$ & 1.100 & 1.100 & Mass & Total & Combined & Wild \\
\hline \multicolumn{11}{|c|}{ PASSERIFORMES } \\
\hline Helmitheros vermivorum & $\begin{array}{l}\text { Vitz \& Rodewald } \\
\text { (2011) }\end{array}$ & $1.00(3)$ & $0.47(2)$ & $0.81(3)$ & 0.810 & 0.381 & Condition & Late & Combined & Wild \\
\hline Seiurus aurocapilla & $\begin{array}{l}\text { Vitz \& Rodewald } \\
\text { (2011) }\end{array}$ & $0.63(3)$ & $0.42(2)$ & $0.84(3)$ & 0.529 & 0.353 & Condition & Late & Combined & Wild \\
\hline Melospiza melodia & $\begin{array}{l}\text { Dybala, Gardali \& } \\
\text { Eadie }(2013)\end{array}$ & $0.14(1)$ & $0.036(1)$ & $1(1)$ & 0.140 & 0.036 & Mass & Total & Combined & Wild \\
\hline funco phaeonotus & Sullivan (1989) & $1.096(2)$ & $0.269(2)$ & $1.125(2)$ & 1.233 & 0.303 & Mass & Late & Combined & Wild \\
\hline \multirow[t]{2}{*}{ Stumella magna } & $\begin{array}{l}\text { Kershner, Walk \& } \\
\text { Warner (2004) }\end{array}$ & $0.175(3)$ & $0.107(2)$ & $4.802(3)$ & 0.840 & 0.514 & Mass & Late & Combined & Wild \\
\hline & $\begin{array}{l}\text { Suedkamp Wells et al. } \\
\quad(2007)\end{array}$ & $0.042(3)$ & $0.032(2)$ & $7.085(3)$ & 0.298 & 0.227 & Mass & Total & Combined & Wild \\
\hline Spiza americana & $\begin{array}{l}\text { Suedkamp Wells et al. } \\
\quad(2007)\end{array}$ & $0.092(3)$ & $0.064(2)$ & $2.62(3)$ & 0.241 & 0.168 & Mass & Total & Combined & Wild \\
\hline Loxops coccineus & $\begin{array}{l}\text { Medeiros \& Freed } \\
\text { (2009) }\end{array}$ & $0.91(3)$ & $0.34(2)$ & $0.88(3)$ & 0.801 & 0.299 & Mass & Late & Combined & Wild \\
\hline \multirow[t]{3}{*}{ Passer domesticus } & Ringsby et al. (1998) & $0.157(1)$ & $0.045(1)$ & $6.25(2)$ & 0.981 & 0.281 & Mass & Late & Combined & Wild \\
\hline & & $0.0942(1)$ & $0.042(1)$ & $6.25(2)$ & 0.589 & 0.263 & Mass & Late & Combined & Wild \\
\hline & Cleasby et al. (2010) & $0.002(1)$ & $0.019(1)$ & $1(1)$ & 0.002 & 0.019 & Mass & Total & Combined & Wild \\
\hline Ficedula albicollis & $\begin{array}{l}\text { Lindén, Gustafsson \& } \\
\text { Part (1992) }\end{array}$ & $0.0231(1)$ & $0.003(1)$ & $1(1)$ & 0.023 & 0.003 & Mass & Late & Combined & Wild \\
\hline \multirow[t]{3}{*}{ Ficedula hypoleuca } & Potti et al. (2002) & $0.43(1)$ & $0.17(1)$ & $1(1)$ & 0.430 & 0.170 & Mass & Total & Female & Wild \\
\hline & & $0.28(1)$ & $0.25(1)$ & $1(1)$ & 0.280 & 0.250 & Mass & Total & Male & Wild \\
\hline & Lobato et al. (2005) & $-0.31(3)$ & $0.36(2)$ & $0.68(3)$ & -0.211 & 0.245 & Mass & Total & Combined & Wild \\
\hline Erythropygia coryphaeus & Lloyd et al. (2009) & $0.39(1)$ & $0.13(1)$ & $2.41(2)$ & 0.940 & 0.313 & Mass & Total & Combined & Wild \\
\hline \multirow[t]{2}{*}{ Turdus merula } & Snow (1958) & $0.011(2)$ & $0.018(2)$ & $8.01(2)$ & 0.088 & 0.144 & Mass & Total & Combined & Wild \\
\hline & Magrath (1991) & $0.076(2)$ & $0.011(2)$ & $7.38(2)$ & 0.561 & 0.081 & Mass & Early & Combined & Wild \\
\hline Hylocichla mustelina & Brown \& Roth (2004) & $0.12(2)$ & $0.077(2)$ & $1(1)$ & 0.120 & 0.077 & Mass & Total & Combined & Wild \\
\hline Cinclus mexicanus & Mackas etal. (2010) & $0.208(2)$ & $0.008(2)$ & $3.447(2)$ & 0.717 & 0.028 & Condition & Total & Combined & Wild \\
\hline Sturnus vulgaris & Serra et al. (2012) & $0.056(3)$ & $0.044(2)$ & $10.8(3)$ & 0.605 & 0.475 & Mass & Early & Female & Wild \\
\hline \multirow[t]{10}{*}{ Parus major } & Garnett (1981) & $0.165(1)$ & $0.0468(1)$ & $1.25(2)$ & 0.206 & 0.059 & Mass & Total & Combined & Wild \\
\hline & $\begin{array}{l}\text { Smith, Kallander \& } \\
\text { Nilsson (1989) }\end{array}$ & $0.347(2)$ & $0.247(2)$ & $1.192(2)$ & 0.414 & 0.294 & Mass & Early & Combined & Wild \\
\hline & Lindén et al. (1992) & $0.0047(1)$ & $0.003(1)$ & $1(1)$ & 0.005 & 0.003 & Mass & Late & Combined & Wild \\
\hline & Verboven \& Visser & $0.135(2)$ & $0.035(2)$ & $1.44(2)$ & 0.194 & 0.050 & Mass & Late & Female & Wild \\
\hline & (1998) & $0.161(2)$ & $0.069(2)$ & $1.444(2)$ & 0.232 & 0.100 & Mass & Late & Female & Wild \\
\hline & & $0.244(2)$ & $0.035(2)$ & $1.518(2)$ & 0.370 & 0.053 & Mass & Late & Male & Wild \\
\hline & & $0.231(2)$ & $0.067(2)$ & $1.494(2)$ & 0.345 & 0.100 & Mass & Late & Male & Wild \\
\hline & $\begin{array}{l}\text { Naef-Daenzer, } \\
\text { Widmer \& Nuber } \\
\text { (2001) }\end{array}$ & $0.045(1)$ & $0.012(1)$ & $15.75(2)$ & 0.709 & 0.189 & Mass & Late & Combined & Wild \\
\hline & $\begin{array}{l}\text { Monrós, Belda \& } \\
\text { Barba (2002) }\end{array}$ & $0.14(1)$ & $0.05(1)$ & $1(1)$ & 0.140 & 0.050 & Mass & Late & Combined & Wild \\
\hline & $\begin{array}{l}\text { Greño, Belda \& Barba } \\
(2008)\end{array}$ & $0.26(1)$ & $0.07(1)$ & $1(1)$ & 0.260 & 0.070 & Mass & Late & Combined & Wild \\
\hline Parus ater & $\begin{array}{l}\text { Naef-Daenzer et al. } \\
\quad(2001)\end{array}$ & $0.045(1)$ & $0.012(1)$ & $15.75(2)$ & 0.709 & 0.189 & Mass & Late & Combined & Wild \\
\hline \multirow[t]{3}{*}{ Parus caeruleus } & Nur (1984) & $0.297(2)$ & $0.13(2)$ & $1.02(2)$ & 0.303 & 0.133 & Mass & Total & Combined & Wild \\
\hline & Raberg, Stjernman \& & $0.295(1)$ & $0.105(1)$ & $1(1)$ & 0.295 & 0.105 & Mass & Total & Male & Wild \\
\hline & Nilsson (2005) & $0.29(1)$ & $0.153(1)$ & $1(1)$ & 0.290 & 0.153 & Mass & Total & Female & Wild \\
\hline Corous frugilegus & $\begin{array}{l}\text { Patterson, Dunnet \& } \\
\text { Goodbody (1988) }\end{array}$ & $0.0084(3)$ & $0.0013(2)$ & $46.8(3)$ & 0.393 & 0.061 & Mass & Late & Combined & Wild \\
\hline
\end{tabular}


Table 2. Continued

\begin{tabular}{|c|c|c|c|c|c|c|c|c|c|c|}
\hline Species & Study & Beta & S.E. & $\begin{array}{l}\text { S.D. } \\
\text { mass }\end{array}$ & $\begin{array}{l}\text { Standardized } \\
\text { beta }\end{array}$ & $\begin{array}{l}\text { Standardized } \\
\text { S.E. }\end{array}$ & $\begin{array}{l}\text { Mass or } \\
\text { condition }\end{array}$ & $\begin{array}{l}\text { Timing of } \\
\text { measurement }\end{array}$ & Sex & $\begin{array}{l}\text { Wild or } \\
\text { captive }\end{array}$ \\
\hline \multirow[t]{2}{*}{ Pica pica } & $\begin{array}{l}\text { Husby \& Slagsvold } \\
\quad(1992)\end{array}$ & $0.03(3)$ & $0.015(2)$ & $33.17(3)$ & 0.995 & 0.498 & Mass & Total & \multicolumn{2}{|c|}{ Combined Wild } \\
\hline & $\begin{array}{l}\text { Ponz Miranda, } \\
\text { Gil-Delgado Alberti } \\
\text { \& López Iborra } \\
(2007)\end{array}$ & $0.039(1)$ & $0.018(1)$ & $22.5(2)$ & 0.765 & 0.360 & Mass & Total & \multicolumn{2}{|c|}{ Combined Wild } \\
\hline \multirow{3}{*}{$\begin{array}{l}\text { Aphelocoma coerulescens } \\
\text { Acanthiza pusilla }\end{array}$} & s Mumme etal. (2015) & $0.069(2)$ & $0.006(2)$ & $6.99(2)$ & 0.482 & 0.042 & Mass & Total & \multicolumn{2}{|c|}{ Combined Wild } \\
\hline & $\begin{array}{l}\text { Green \& Cockburn } \\
\quad(2001)\end{array}$ & $1.7(2)$ & $0.783(2)$ & $0.35(2)$ & 0.595 & 0.274 & Mass & Total & Male & Wild \\
\hline & & $0.661(2)$ & $0.562(2)$ & $0.43(2)$ & 0.284 & 0.242 & Mass & Total & Female & Wild \\
\hline \multirow[t]{2}{*}{ Tyrannus tyrannus } & Dolan et al. (2009) & $0.022(1)$ & $0.062(1)$ & $2.483(1)$ & 0.055 & 0.154 & Mass & Total & Male & Wild \\
\hline & & $-0.076(1)$ & $0.08(1)$ & $2.5(1)$ & -0.190 & 0.200 & Mass & Total & Female & Wild \\
\hline Platycercus elagans & Krebs (1999) & $0.138(3)$ & $0.162(2)$ & $2.12(3)$ & 0.293 & 0.343 & Mass & Total & Male & Wild \\
\hline Clamator glandarius & $\begin{array}{r}\text { Soler, Palomino \& } \\
\text { Martinez (1994) }\end{array}$ & $0.187(3)$ & $0.067(2)$ & $11.45(3)$ & 2.141 & 0.767 & Mass & Late & \multicolumn{2}{|c|}{ Combined Wild } \\
\hline \multicolumn{11}{|c|}{ GHARADRIIFORMES } \\
\hline Fratercula cirrhata & Morrison et al. (2009) & $0.0012(1)$ & $0.0039(1)$ & $62.5(2)$ & 0.075 & 0.244 & Mass & Late & \multirow{2}{*}{\multicolumn{2}{|c|}{$\begin{array}{l}\text { Combined Wild } \\
\text { Combined Wild }\end{array}$}} \\
\hline Fratercula arctica & $\begin{array}{l}\text { Harris \& Rothery } \\
\text { (1985) }\end{array}$ & $-0.002(3)$ & $0.0054(2)$ & $27.456(3)$ & -0.055 & 0.148 & Mass & Late & & \\
\hline Uria aalge & Hedgren (1981) & $-0.002(2)$ & $0.002(2)$ & $24.87(2)$ & -0.050 & 0.050 & Mass & Late & \multirow{4}{*}{\multicolumn{2}{|c|}{$\begin{array}{l}\text { Combined Wild } \\
\text { Combined Wild } \\
\text { Combined Wild } \\
\text { Combined Wild }\end{array}$}} \\
\hline \multirow[t]{2}{*}{ Alca torda } & Lloyd (1979) & $0.11(3)$ & $0.07(2)$ & $6.15(3)$ & 0.677 & 0.431 & Mass & Total & & \\
\hline & & $0.19(3)$ & $0.11(2)$ & $6.45(3)$ & 1.226 & 0.710 & Mass & Total & & \\
\hline $\begin{array}{l}\text { Synthliboramphus } \\
\text { antiquus }\end{array}$ & Gaston (1997) & $0.239(1)$ & $0.103(1)$ & $2(3)$ & 0.478 & 0.206 & Mass & Late & & \\
\hline Sterna dougallii & $\begin{array}{l}\text { Monticelli \& Ramos } \\
\text { (2012) }\end{array}$ & $0.52(3)$ & $0.24(2)$ & $0.47(3)$ & 0.244 & 0.113 & Condition & Total & \multicolumn{2}{|c|}{ Combined Wild } \\
\hline \multirow[t]{3}{*}{ Sterna hirundo } & $\begin{array}{l}\text { Schauroth \& Becker } \\
\text { (2008) }\end{array}$ & $0.19(3)$ & $0.07(2)$ & $13.3(3)$ & 2.527 & 0.931 & Mass & Late & Male & Wild \\
\hline & & $0.1(3)$ & $0.06(2)$ & $10.5(3)$ & 1.050 & 0.630 & Mass & Late & Female & Wild \\
\hline & $\begin{array}{l}\text { Braasch, Schauroth \& } \\
\text { Becker }(2009)\end{array}$ & $0.023(1)$ & $0.014(1)$ & $12.506(3)$ & 0.288 & 0.175 & Mass & Late & \multicolumn{2}{|c|}{ Combined Wild } \\
\hline Sterna sandvicensis & $\begin{array}{l}\text { Stienen \& } \\
\text { Brenninkmeijer } \\
(2002)\end{array}$ & $-0.80(3)$ & $1.11(2)$ & $0.184(3)$ & -0.147 & 0.204 & Condition & Late & \multicolumn{2}{|c|}{ Combined Wild } \\
\hline Larus fuscus & Bolton (1991) & $0.081(3)$ & $0.044(2)$ & $6.41(3)$ & 0.519 & 0.282 & Mass & Early & \multirow{2}{*}{\multicolumn{2}{|c|}{$\begin{array}{l}\text { Combined Wild } \\
\text { Combined Wild }\end{array}$}} \\
\hline Haemotopus ostralegus & $\begin{array}{l}\text { Kersten \& } \\
\text { Brenninkmeijer } \\
\text { (1995) }\end{array}$ & $0.0065(1)$ & $0.005(1)$ & $27.5(2)$ & 0.179 & 0.138 & Mass & Late & & \\
\hline Charadrius montanus & $\begin{array}{l}\text { Dinsmore, White \& } \\
\text { Knopf (2003) }\end{array}$ & $0.77(1)$ & $0.265(1)$ & $1(1)$ & 0.77 & 0.265 & Mass & Total & \multicolumn{2}{|c|}{ Combined Wild } \\
\hline \multicolumn{11}{|l|}{ OTIDIFORMES } \\
\hline \multirow[t]{2}{*}{ Otis tarda } & Martín etal. (2007) & $0.0012(2)$ & $0.00035(2)$ & $500(2)$ & 0.600 & 0.175 & Mass & Late & Male & Wild \\
\hline & & $0.0012(2)$ & $0.0004(2)$ & $500(2)$ & 0.600 & 0.200 & Mass & Late & Female & Wild \\
\hline \multicolumn{11}{|c|}{ SPHENISCIFORMES } \\
\hline Eudyptes chrysolophus & Horswill et al. (2014) & $0.41(1)$ & $0.18(1)$ & $1(1)$ & 0.410 & 0.180 & Mass & Late & \multirow{4}{*}{\multicolumn{2}{|c|}{$\begin{array}{l}\text { Combined Wild } \\
\text { Combined Wild } \\
\text { Combined Wild } \\
\text { Combined Wild }\end{array}$}} \\
\hline Megadyptes antipodes & McClung et al. (2004) & $0.51(1)$ & $0.072(1)$ & $0.594(3)$ & 0.303 & 0.043 & Mass & Late & & \\
\hline Aptenodytes patagonicus & Olsson (1997) & $0.074(3)$ & $0.17(2)$ & $2.29(3)$ & 0.169 & 0.389 & Mass & Total & & \\
\hline Pygoscelis papua & $\begin{array}{l}\text { Williams \& Croxall } \\
\text { (1991) }\end{array}$ & $0.007(3)$ & $0.003(2)$ & $132(3)$ & 0.924 & 0.396 & Mass & Late & & \\
\hline \multicolumn{11}{|c|}{ PROCELLARIIFORMES } \\
\hline Puffinus puffinus & $\begin{array}{l}\text { Perrins, Harris \& } \\
\text { Britton (1973) }\end{array}$ & $0.011(3)$ & $0.003(2)$ & $63.38(3)$ & 0.697 & 0.190 & Mass & Late & Combined & Wild \\
\hline Puffinus griseus & $\begin{array}{l}\text { Sagar \& Horning } \\
\quad(1998)\end{array}$ & $0.0034(3)$ & $0.0012(2)$ & $166.3(3)$ & 0.565 & 0.200 & Mass & Late & Combined & Wild \\
\hline Diomedea exulans & $\begin{array}{l}\text { Weimerskirch, } \\
\text { Barbraud \& Lys } \\
(2000)\end{array}$ & $0.004(3)$ & $0.005(2)$ & $60.5(1)$ & 0.242 & 0.303 & Mass & Total & Male & Wild \\
\hline & & $0.075(3)$ & $0.039(2)$ & $32(1)$ & 2.400 & 1.248 & Mass & Total & Female & Wild \\
\hline SULIFORMES & & & & & & & & & & \\
\hline Sula granti & $\begin{array}{l}\text { Maness \& Anderson } \\
\quad(2013)\end{array}$ & $0.000247(2)$ & 0.000219 & $236.702(1)$ & 0.058 & 0.052 & Mass & Late & Male & Wild \\
\hline & & $0.000261(2)$ & $0.000264(2)$ & $234.526(1)$ & 0.061 & 0.062 & Mass & Late & Female & Wild \\
\hline ANSERIFORMES & & & & & & & & & & \\
\hline Aythya valisineria & $\begin{array}{l}\text { Anderson, Lindberg } \\
\text { \& Emery (2001) }\end{array}$ & $0.113(1)$ & $0.091(1)$ & $0.165(2)$ & 0.019 & 0.015 & Mass & Total & Combined & Wild \\
\hline Aythya affinis & $\begin{array}{l}\text { Rotella, Clark \& } \\
\text { Afron (2003) }\end{array}$ & $0.45(1)$ & $0.24(1)$ & $1(1)$ & 0.450 & 0.240 & Mass & Total & Female & Wild \\
\hline
\end{tabular}


Table 2. Continued

\begin{tabular}{|c|c|c|c|c|c|c|c|c|c|c|}
\hline Species & Study & Beta & S.E. & $\begin{array}{l}\text { S.D. } \\
\text { mass }\end{array}$ & $\begin{array}{l}\text { Standardized } \\
\text { beta }\end{array}$ & $\begin{array}{l}\text { Standardized } \\
\text { S.E. }\end{array}$ & $\begin{array}{l}\text { Mass or } \\
\text { condition }\end{array}$ & $\begin{array}{c}\text { Timing of } \\
\text { measurement }\end{array}$ & Sex & $\begin{array}{l}\text { Wild or } \\
\text { captive }\end{array}$ \\
\hline Aix sponsa & Davis et al. (2007) & $0.083(1)$ & $0.031(1)$ & $1.5(2)$ & 0.125 & 0.047 & Mass & Early & Combined & Wild \\
\hline Melanitta fusca & $\begin{array}{l}\text { Traylor \& Alisauskas } \\
\quad(2006)\end{array}$ & $0.2(1)$ & $0.077(1)$ & $1(1)$ & 0.200 & 0.077 & Condition & Early & Combined & Wild \\
\hline \multirow[t]{2}{*}{ Chen caerulescens } & Cooch (2002) & $0.004(2)$ & $0.001(2)$ & $78(3)$ & 0.312 & 0.078 & Mass & Total & Female & Wild \\
\hline & $\begin{array}{l}\text { Souchay, Gauthier \& } \\
\text { Pradel (2013) }\end{array}$ & $1.66(1)$ & $0.898(1)$ & $0.5(2)$ & 0.830 & 0.449 & Condition & Late & Female & Wild \\
\hline \multirow[t]{2}{*}{ Chen canagica } & Schmutz (1993) & $0.002(3)$ & $0.001(2)$ & $122.7(3)$ & 0.245 & 0.123 & Mass & Total & Female & Wild \\
\hline & & $0.002(3)$ & $0.00084(2)$ & $143.2(3)$ & 0.286 & 0.120 & Mass & Total & Male & Wild \\
\hline \multirow[t]{2}{*}{ Branta leucopsis } & Owen \& Black (1989) & $0.005(3)$ & $0.001(2)$ & $166.5(3)$ & 0.833 & 0.167 & Mass & Late & Combined & Wild \\
\hline & $\begin{array}{l}\text { Van der Jeugd \& } \\
\text { Larsson (1998) }\end{array}$ & $0.00176(2)$ & $0.000832(2)$ & $211.7(1)$ & 0.373 & 0.176 & Mass & Late & Combined & Wild \\
\hline \multicolumn{11}{|l|}{ GALLIFORMES } \\
\hline Colinus virginianus & Lusk et al. (2005) & $0.014(2)$ & $0.002(2)$ & $47.57(2)$ & 0.666 & 0.095 & Mass & Early & Combined & Wild \\
\hline
\end{tabular}

being captive when the individuals were kept in an enclosure and artificially fed. Captive animals do not display the same mortality patterns as free-ranging animals (e.g. Lemaitre etal., 2013; Tidière etal., 2016). In particular, captive individuals have access to veterinary care that can markedly influence the magnitude of the offspring mass - survival relationship. As all bird populations included in the meta-analysis were free-living, we only tested an effect of captive versus wild conditions in mammals.

(4) The occurrence of predation in the studied population was implemented as a two-level factor (Predation versus No predation) for the analysis of the relationship between offspring survival and body mass. We first considered the information provided in the paper about the occurrence of predation. When no information about the causes of mortality was reported, we searched for other papers about the same population to find out whether the focal population was subjected to predation. We expected that predation should decrease the effect of body mass on offspring survival because predators generally prey upon juveniles independently from their mass (Hurley etal., 2011; Keech etal., 2011). This moderator was only tested for mammalian populations because all of the bird populations included in our data set were subjected to predation. We did not report any information about hunting in populations because juveniles are typically not hunted.

(5) Offspring sex was included as a three-level factor (Female, Male or Combined). 'Combined' corresponds to studies in which individuals from both sexes were pooled within the same relationship. 'Male' and 'Female' correspond to studies in which sex-specific relationships were provided. We looked for potential sex differences in the effect sizes of the relationships.

(6) The influence of species-specific mating system was tested differently in mammals and birds. As only two mating systems occurred in our set of mammalian species, we included this moderator as a two-level factor (Polygynous versus Promiscuous). Our bird species were principally socially monogamous, although a high rate of extra-pair paternities occurred in several species (Garamszegi etal., 2005). As the degree of monogamy can impact the amount of parental care and thereby influence the offspring mass-survival relationship (Jašarević et al., 2013) we distinguished between strict monogamy and other mating systems. We defined species as being strictly monogamous when the rate of extra-pair paternities was lower than $5 \%$. As the magnitude of between-sex differences might differ in relation to mating systems, we included a test of the interaction between mating system and sex in our analyses.

(7) Litter size was implemented as a two-level factor in mammals (Monotocous versus Polytocous). Monotocous species have a mean litter size of one, whereas polytocous species produce more than one offspring per litter. Brood size in birds was measured as the average clutch size for each species (i.e. a continuous variable). Litter (mammals) or clutch (birds) size could influence the offspring mass-survival relationship because of the expected offspring size-number trade-off (Smith \& Fretwell, 1974). We also tested for the interaction between litter size and mating system for mammals because siblings in species displaying a promiscuous mating system are expected to face higher sibling competition than siblings in species with other mating systems (Forstmeier etal., 2014; Garratt etal., 2014).

(8) Data quality was implemented as a two-level factor (High quality versus Low quality). Data were considered as high quality when all data required for the analysis were explicitly reported. Low-quality data corresponded to case studies for which the required data were extracted from graphs or obtained from simulations. We thus tested whether the data-extraction 
Table 3. Summary of the statistics and potential driving factors compiled in the meta-analysis for the relationship between offspring mass and maternal mass in mammals. Pearson correlation coefficient and sample size are reported. The extraction procedure is reported in parentheses as 1 if the statistic is directly calculated in the study, and 2 if data are reported from a figure. See Section $2.3 b$ for more information on the extraction procedure and Section II.4b for explanation of Mass or other relationships and Offspring age categories

\begin{tabular}{|c|c|c|c|c|c|c|c|c|c|}
\hline Species & Study & Pearson's $r$ & $\mathcal{N}$ & Fisher $Z_{r}$ & S.E. & $\begin{array}{l}\text { Mass or other } \\
\text { relationships }\end{array}$ & Offspring age & Sex & $\begin{array}{l}\text { Wild or } \\
\text { captive }\end{array}$ \\
\hline \multicolumn{10}{|c|}{ DASYUROMORPHIA } \\
\hline \multirow[t]{2}{*}{ Phascogale calura } & Foster \& Taggart & $0.494(2)$ & 16 & 0.541 & 0.277 & Mass & Weaning & Male & Captive \\
\hline & (2008) & $0.554(2)$ & 13 & 0.624 & 0.316 & Mass & Weaning & Female & Captive \\
\hline \multicolumn{10}{|c|}{ DIPROTODONTIA } \\
\hline \multirow[t]{2}{*}{ Phascolarctos cinereus } & Tobey et al. (2006) & $0.259(1)$ & 27 & 0.265 & 0.204 & Mass & Weaning & Female & Captive \\
\hline & & $0.298(1)$ & 27 & 0.307 & 0.204 & Mass & Weaning & Male & Captive \\
\hline \multicolumn{10}{|l|}{ GHIROPTERA } \\
\hline \multirow[t]{2}{*}{ Eptesicus fuscus } & Booher (2008) & $0.458(1)$ & 10 & 0.495 & 0.378 & Mass & Birth & Combined & Captive \\
\hline & & $0.854(1)$ & 9 & 1.271 & 0.408 & Mass & Birth & Combined & Captive \\
\hline \multicolumn{10}{|l|}{ GARNIVORA } \\
\hline Mungo mungo & Hodge et al. (2009) & $0.481(2)$ & 39 & 0.524 & 0.167 & Mass & Birth & Combined & Wild \\
\hline Suricata suricatta & Russell et al. (2003) & $0.469(1)$ & 37 & 0.509 & 0.171 & Mass & Weaning & Combined & Wild \\
\hline Ursus maritimus & $\begin{array}{l}\text { Derocher \& Stirling } \\
\quad(1994)\end{array}$ & $0.84(1)$ & 27 & 1.221 & 0.204 & Mass & Birth & Combined & Wild \\
\hline \multirow[t]{4}{*}{ Ursus arctos } & $\begin{array}{l}\text { Noyce, Coy \& } \\
\quad \text { Garshelis (2002) }\end{array}$ & $0.624(1)$ & 59 & 0.732 & 0.134 & Mass & Birth & Combined & Wild \\
\hline & Dahle et al. (2006) & $0.232(1)$ & 224 & 0.236 & 0.067 & Mass & Birth & Combined & Wild \\
\hline & Gonzalez et al. (2012) & $0.173(1)$ & 254 & 0.175 & 0.063 & Other & Birth & Combined & Wild \\
\hline & Robbins et al. (2012) & $0.775(1)$ & 18 & 1.033 & 0.258 & Mass & Birth & Combined & Wild \\
\hline \multirow[t]{6}{*}{ Mirounga leonina } & $\begin{array}{c}\text { McCann, Fedak \& } \\
\text { Harwood (1989) }\end{array}$ & $0.812(1)$ & 13 & 1.133 & 0.316 & Mass & Birth & Female & Wild \\
\hline & & $0.332(1)$ & 16 & 0.345 & 0.277 & Mass & Birth & Male & Wild \\
\hline & $\begin{array}{l}\text { Fedak, Arnbom \& } \\
\text { Boyd (1996) }\end{array}$ & $0.701(1)$ & 12 & 0.869 & 0.333 & Mass & Birth & Male & Wild \\
\hline & & $0.552(1)$ & 15 & 0.621 & 0.289 & Mass & Birth & Female & Wild \\
\hline & Arnbom et al. (1997) & $0.146(2)$ & 74 & 0.147 & 0.119 & Mass & Birth & Female & Wild \\
\hline & & $0.141(2)$ & 63 & 0.142 & 0.129 & Mass & Birth & Male & Wild \\
\hline $\begin{array}{l}\text { Mirounga } \\
\text { angustirostris }\end{array}$ & Crocker et al. (2001) & $0.57(1)$ & 16 & 0.648 & 0.277 & Other & Weaning & Combined & Wild \\
\hline Leptonychotes weddellii & Wheatley et al. (2006) & $0.768(2)$ & 47 & 1.015 & 0.151 & Mass & Weaning & Combined & Wild \\
\hline \multirow[t]{7}{*}{ Phoca vitulina } & Bowen et al. (1994) & $0.42(1)$ & 124 & 0.448 & 0.091 & Mass & Birth & Female & Wild \\
\hline & & $0.32(1)$ & 134 & 0.332 & 0.087 & Mass & Birth & Male & Wild \\
\hline & Coltman et al. (1998) & $0.13(1)$ & 60 & 0.131 & 0.132 & Mass & Birth & Combined & Wild \\
\hline & Ellis et al. (2000) & $0.41(1)$ & 118 & 0.436 & 0.093 & Mass & Birth & Male & Wild \\
\hline & & $0.5(1)$ & 126 & 0.549 & 0.090 & Mass & Birth & Female & Wild \\
\hline & Bowen et al. $(2001 a)$ & $0.51(1)$ & 100 & 0.563 & 0.102 & Mass & Weaning & Combined & Wild \\
\hline & Bowen et al. $(2001 b)$ & $0.28(1)$ & 30 & 0.288 & 0.192 & Mass & Birth & Combined & Wild \\
\hline \multirow[t]{2}{*}{ Halichoerus grypus } & Iverson et al. (1993) & $0.567(2)$ & 9 & 0.643 & 0.408 & Mass & Birth & Combined & Wild \\
\hline & Pomeroy et al. (1999) & $0.316(1)$ & 95 & 0.327 & 0.104 & Mass & Birth & Combined & Wild \\
\hline Phocarctos hookeri & Chilvers et al. (2007) & $0.543(1)$ & 98 & 0.608 & 0.103 & Mass & Birth & Combined & Wild \\
\hline \multirow{9}{*}{ Arctocephalus gazella } & Boyd \& McCann & $0.028(2)$ & 35 & 0.028 & 0.177 & Mass & Birth & Female & Wild \\
\hline & (1989) & $0.42(1)$ & 40 & 0.448 & 0.164 & Mass & Birth & Male & Wild \\
\hline & Lunn \& Boyd (1993) & $0.09(1)$ & 32 & 0.090 & 0.186 & Mass & Birth & Male & Wild \\
\hline & & $0.251(1)$ & 17 & 0.256 & 0.267 & Mass & Birth & Male & Wild \\
\hline & & $0.597(1)$ & 14 & 0.688 & 0.302 & Mass & Birth & Male & Wild \\
\hline & & $0.386(1)$ & 54 & 0.407 & 0.140 & Mass & Birth & Female & Wild \\
\hline & & $0.637(1)$ & 17 & 0.753 & 0.267 & Mass & Birth & Female & Wild \\
\hline & & $0.162(1)$ & 19 & 0.163 & 0.250 & Mass & Birth & Female & Wild \\
\hline & McDonald et al. (2012) & $0.469(1)$ & 49 & 0.509 & 0.147 & Mass & Birth & Combined & Wild \\
\hline \multirow[t]{2}{*}{ Callorhinus ursinus } & Boltnev \& York (2001) & $0.287(2)$ & 137 & 0.295 & 0.086 & Mass & Birth & Female & Wild \\
\hline & & $0.329(2)$ & 106 & 0.342 & 0.099 & Mass & Birth & Male & Wild \\
\hline \multicolumn{10}{|l|}{ ARTIODAGTYLA } \\
\hline Dama dama & $\begin{array}{l}\text { Birgersson \& Ekvall } \\
\quad(1997)\end{array}$ & $0.61(1)$ & 138 & 0.709 & 0.086 & Mass & Birth & Combined & Captive \\
\hline
\end{tabular}


Table 3. Continued

\begin{tabular}{|c|c|c|c|c|c|c|c|c|c|}
\hline Species & Study & Pearson's $r$ & $\mathcal{N}$ & Fisher $Z_{r}$ & S.E. & $\begin{array}{l}\text { Mass or other } \\
\text { relationships }\end{array}$ & Offspring age & Sex & $\begin{array}{l}\text { Wild or } \\
\text { captive }\end{array}$ \\
\hline \multirow[t]{5}{*}{ Cervus elaphus } & $\begin{array}{l}\text { Clutton-Brock, Albon } \\
\text { \& Guinness (1986) }\end{array}$ & $0.455(1)$ & 104 & 0.491 & 0.100 & Mass & Birth & Combined & Wild \\
\hline & $\begin{array}{l}\text { Moore, Littlejohn \& } \\
\text { Cowie (1988) }\end{array}$ & $0.437(1)$ & 143 & 0.469 & 0.085 & Mass & Birth & Combined & Captive \\
\hline & Bonenfant et al. (2003) & $0.436(1)$ & 46 & 0.467 & 0.152 & Mass & Weaning & Combined & Wild \\
\hline & $\begin{array}{l}\text { Landete-Castillejos } \\
\text { et al. }(2003)\end{array}$ & $0.39(1)$ & 24 & 0.412 & 0.218 & Mass & Birth & Combined & Captive \\
\hline & $\begin{array}{l}\text { Landete-Castillejos } \\
\quad \text { et al. (2005) }\end{array}$ & $0.46(1)$ & 91 & 0.497 & 0.107 & Mass & Birth & Combined & Captive \\
\hline Odocoileus virginianus & Michel et al. (2015) & $0.318(1)$ & 229 & 0.329 & 0.067 & Mass & Birth & Combined & Captive \\
\hline \multirow[t]{10}{*}{ Rangifer tarandus } & Rognmo et al. (1983) & $0.752(2)$ & 39 & 0.978 & 0.167 & Mass & Birth & Combined & Captive \\
\hline & $\begin{array}{l}\text { Eloranta \& Nieminen } \\
\quad(1986)\end{array}$ & $0.58(1)$ & 70 & 0.662 & 0.122 & Mass & Birth & Combined & Captive \\
\hline & Kojola (1993) & $0.656(1)$ & 65 & 0.786 & 0.127 & Mass & Weaning & Female & Captive \\
\hline & & $0.657(1)$ & 55 & 0.788 & 0.139 & Mass & Weaning & Male & Captive \\
\hline & Holand et al. (2004) & $0.607(1)$ & 52 & 0.704 & 0.143 & Mass & Birth & Combined & Captive \\
\hline & Adams (2005) & $0.47(1)$ & 46 & 0.510 & 0.152 & Mass & Birth & Combined & Wild \\
\hline & Holand et al. (2006) & $0.249(1)$ & 66 & 0.254 & 0.126 & Mass & Birth & Combined & Captive \\
\hline & Mysterud et al. (2009) & $0.29(1)$ & 88 & 0.299 & 0.108 & Mass & Birth & Combined & Captive \\
\hline & Taillon et al. (2012) & $0.55(2)$ & 48 & 0.618 & 0.149 & Mass & Birth & Combined & Wild \\
\hline & & $0.272(2)$ & 48 & 0.279 & 0.149 & Mass & Birth & Combined & Wild \\
\hline \multirow[t]{4}{*}{ Capreolus capreolus } & Hewison et al. (2005) & $0.476(1)$ & 35 & 0.518 & 0.177 & Mass & Weaning & Female & Wild \\
\hline & & $0.259(1)$ & 38 & 0.265 & 0.169 & Mass & Weaning & Male & Wild \\
\hline & & $0.482(1)$ & 38 & 0.526 & 0.169 & Mass & Weaning & Female & Wild \\
\hline & & $0.366(1)$ & 36 & 0.384 & 0.174 & Mass & Weaning & Male & Wild \\
\hline Alces alces & Keech et al. (2000) & $0.458(1)$ & 37 & 0.495 & 0.171 & Mass & Birth & Combined & Wild \\
\hline \multirow[t]{2}{*}{ Bison bison } & Hamel et al. $(2012 a)$ & $0.374(2)$ & 316 & 0.393 & 0.057 & Mass & Weaning & Male & Wild \\
\hline & & $0.267(2)$ & 302 & 0.274 & 0.058 & Mass & Weaning & Female & Wild \\
\hline Oreamnos americanus & $\begin{array}{l}\text { Côté \& Festa-Bianchet } \\
\text { (2001) }\end{array}$ & $0.412(1)$ & 32 & 0.438 & 0.186 & Mass & Birth & Combined & Wild \\
\hline Ovis canadensis & $\begin{array}{l}\text { Festa-Bianchet \& } \\
\text { Jorgenson (1998) }\end{array}$ & $0.289(1)$ & 231 & 0.297 & 0.066 & Mass & Weaning & Combined & Wild \\
\hline \multirow[t]{2}{*}{ Ovis aries } & $\begin{array}{l}\text { Clutton-Brock et al. } \\
\text { (1996) }\end{array}$ & $0.266(1)$ & 350 & 0.273 & 0.054 & Mass & Birth & Combined & Wild \\
\hline & Steinheim et al. (2002) & $0.045(1)$ & 120000 & 0.045 & 0.003 & Mass & Weaning & Combined & Captive \\
\hline \multicolumn{10}{|l|}{ PRIMATES } \\
\hline Macaca mulatta & $\begin{array}{l}\text { Bercovitch, Widdig \& } \\
\text { Nürnberg }(2000)\end{array}$ & $0.289(1)$ & 97 & 0.297 & 0.103 & Mass & Weaning & Combined & Wild \\
\hline & \multicolumn{9}{|c|}{ RODENTIA } \\
\hline \multirow[t]{2}{*}{ Sciurus vulgaris } & Wauters et al. (1993) & $0.49(1)$ & 57 & 0.536 & 0.136 & Mass & Weaning & Combined & Wild \\
\hline & & $0.64(1)$ & 28 & 0.758 & 0.200 & Mass & Weaning & Combined & Wild \\
\hline \multirow[t]{2}{*}{$\begin{array}{l}\text { Spermophilus } \\
\text { richardsonii }\end{array}$} & $\begin{array}{l}\text { Dobson \& Michener } \\
\quad(1995)\end{array}$ & $0.31(1)$ & 51 & 0.321 & 0.144 & Other & Birth & Combined & Wild \\
\hline & & $0.53(1)$ & 38 & 0.590 & 0.169 & Other & Birth & Combined & Wild \\
\hline \multirow[t]{3}{*}{$\begin{array}{l}\text { Spermophilus } \\
\text { columbianus }\end{array}$} & $\begin{array}{l}\text { Skibiel, Dobson \& } \\
\text { Murie (2009) }\end{array}$ & $0.37(1)$ & 66 & 0.388 & 0.126 & Other & Weaning & Combined & Wild \\
\hline & & $0.34(1)$ & 28 & 0.354 & 0.200 & Other & Weaning & Combined & Wild \\
\hline & & $0.37(1)$ & 93 & 0.388 & 0.105 & Other & Weaning & Combined & Wild \\
\hline \multirow[t]{2}{*}{ Marmota flaviventris } & Monclús et al. (2014) & $0.253(2)$ & 82 & 0.259 & 0.113 & Mass & Weaning & Combined & Wild \\
\hline & & $-0.183(2)$ & 70 & -0.185 & 0.122 & Mass & Weaning & Combined & Wild \\
\hline \multirow[t]{4}{*}{ Cavia aperea } & $\begin{array}{c}\text { Kasparian, Geißler \& } \\
\text { Trillmich (2005) }\end{array}$ & $0.37(1)$ & 81 & 0.388 & 0.113 & Mass & Birth & Combined & Captive \\
\hline & & $0.184(1)$ & 117 & 0.186 & 0.094 & Mass & Birth & Combined & Captive \\
\hline & & $0.464(1)$ & 35 & 0.502 & 0.177 & Mass & Birth & Combined & Captive \\
\hline & & $0.335(1)$ & 10 & 0.348 & 0.378 & Mass & Birth & Combined & Captive \\
\hline $\begin{array}{l}\text { Microtus } \\
\quad \text { pennsylvanicus }\end{array}$ & $\begin{array}{l}\text { Dobson \& Myers } \\
\text { (1989) }\end{array}$ & $0.11(1)$ & 135 & 0.110 & 0.087 & Mass & Birth & Combined & Captive \\
\hline \multirow[t]{2}{*}{ Microtus agrestis } & Koskela et al. (2004) & $0.218(2)$ & 83 & 0.222 & 0.112 & Other & Birth & Male & Captive \\
\hline & & $0.419(2)$ & 88 & 0.446 & 0.108 & Other & Birth & Female & Captive \\
\hline
\end{tabular}


Table 3. Continued

\begin{tabular}{|c|c|c|c|c|c|c|c|c|c|}
\hline Species & Study & Pearson's $r$ & $\mathcal{N}$ & Fisher $z_{r}$ & S.E. & $\begin{array}{l}\text { Mass or other } \\
\text { relationships }\end{array}$ & Offspring age & Sex & $\begin{array}{l}\text { Wild or } \\
\text { captive }\end{array}$ \\
\hline & $\begin{array}{l}\text { Helle, Laaksonen \& } \\
\text { Huitu (2013) }\end{array}$ & $0.066(1)$ & 67 & 0.066 & 0.125 & Mass & Birth & Female & Captive \\
\hline & & $0.479(1)$ & 53 & 0.522 & 0.141 & Mass & Birth & Male & Captive \\
\hline $\begin{array}{l}\text { Peromyscus } \\
\quad \text { maniculatus }\end{array}$ & Myers \& Master (1983) & $0.245(1)$ & 393 & 0.250 & 0.051 & Mass & Birth & Combined & Captive \\
\hline Sigmodon hispidus & $\begin{array}{l}\text { Campbell \& Slade } \\
\text { (1995) }\end{array}$ & $0.48(1)$ & 29 & 0.523 & 0.196 & Mass & Birth & Combined & Captive \\
\hline \multirow[t]{2}{*}{ Mus musculus } & Krackow (1997) & $0.506(1)$ & 83 & 0.557 & 0.112 & Mass & Weaning & Male & Captive \\
\hline & & $0.479(1)$ & 71 & 0.522 & 0.121 & Mass & Weaning & Female & Captive \\
\hline \multirow[t]{2}{*}{ Apodemus argentus } & $\begin{array}{l}\text { Shibata \& Kawamichi } \\
\text { (2009) }\end{array}$ & $0.292(2)$ & 53 & 0.301 & 0.141 & Mass & Birth & Male & Wild \\
\hline & & $0.197(2)$ & 58 & 0.200 & 0.135 & Mass & Birth & Female & Wild \\
\hline
\end{tabular}

procedure had any detectable impact on the results.

To assess the impact of these different moderators on the relationships of interest, we reported the mean difference between the groups with the $95 \%$ highest posterior density interval (the odds ratios were log-transformed to obtain a meaningful mean difference between groups). The mean difference was considered as statistically significant when 0 did not fall within the credibility interval.

\section{(5) Publication bias}

If studies with no detectable effects are less likely to be published (Rosenthal, 1979), the meta-analysis performed from published information would lead to an overestimate of the true effect. To test whether such a publication bias was present in our data, funnel plots were built. The standard diagram plots the precision of the study (measured as the inverse of the standard error) against the mean of the study (Egger et al., 1997). The closer the mean is to the meta-analysis mean, the greater the precision. In the absence of any bias the diagram should be perfectly symmetrical around the mean. To test the symmetry of the diagram a linear regression of the means of each study as a function of their precision is performed. This test is known as the Egger regression (Egger et al., 1997). However, the means are not independent from each other, leading a key assumption of linear regression to be violated. The only values that were independent between the different effect sizes were the residuals of the meta-analysis (Nakagawa \& Santos, 2012), which correspond to the variance that is not explained by the different random factors. The residuals should be symmetrically distributed around 0 . A linear regression of residuals on the precision of the study was performed. A publication bias occurs when the intercept of the regression is statistically different from 0 . To assess the influence of publication bias, the trim and fill method of the package metafor (Viechtbauer, 2010) was used. This method provides an estimate of the number of studies that are absent on one side of the funnel plot and adjusts the meta-analysis mean accordingly. It should not be interpreted as an exact correcting factor of the publication bias but rather provides an assessment of the magnitude of the publication bias.

\section{RESULTS}

\section{(1) Relationship between offspring mass and offspring survival}

\section{(a) Data set}

In mammals, we extracted 75 effect sizes from 60 published papers. These effect sizes came from 33 different species. Overall, Carnivora (9 species), Artiodactyla (15 species) and Rodentia (6 species) were the most represented mammalian orders (Table 1; Fig. 2A). In birds, we extracted 86 effect sizes from 58 published studies. These effect sizes corresponded to 56 different species, mostly Passeriformes (25 species), Charadriiformes (11 species) and Anseriformes (7 species) (Table 2; Fig. 2B).

\section{(b) Results from general meta-analyses}

In mammals, offspring mass positively influenced offspring survival with a meta-analysis mean of 1.82. This effect was statistically significant because the highest posterior density interval of the odds ratio did not overlap 1 [HPDI $=(1.37$; 2.41)] (Fig. 3A). In birds, the same positive effect of mass occurred for offspring survival (meta-analysis mean $=1.48$ ). This effect was also statistically significant $[\mathrm{HPDI}=(1.26$; 1.72)] (Fig. 3B).

The heterogeneity analysis in mammals indicated that each random effect (the effect of phylogeny, of species independently of phylogeny, of population and of first author) included in our meta-analysis only accounted for a weak but similar proportion of heterogeneity among studies, with an 
(A)

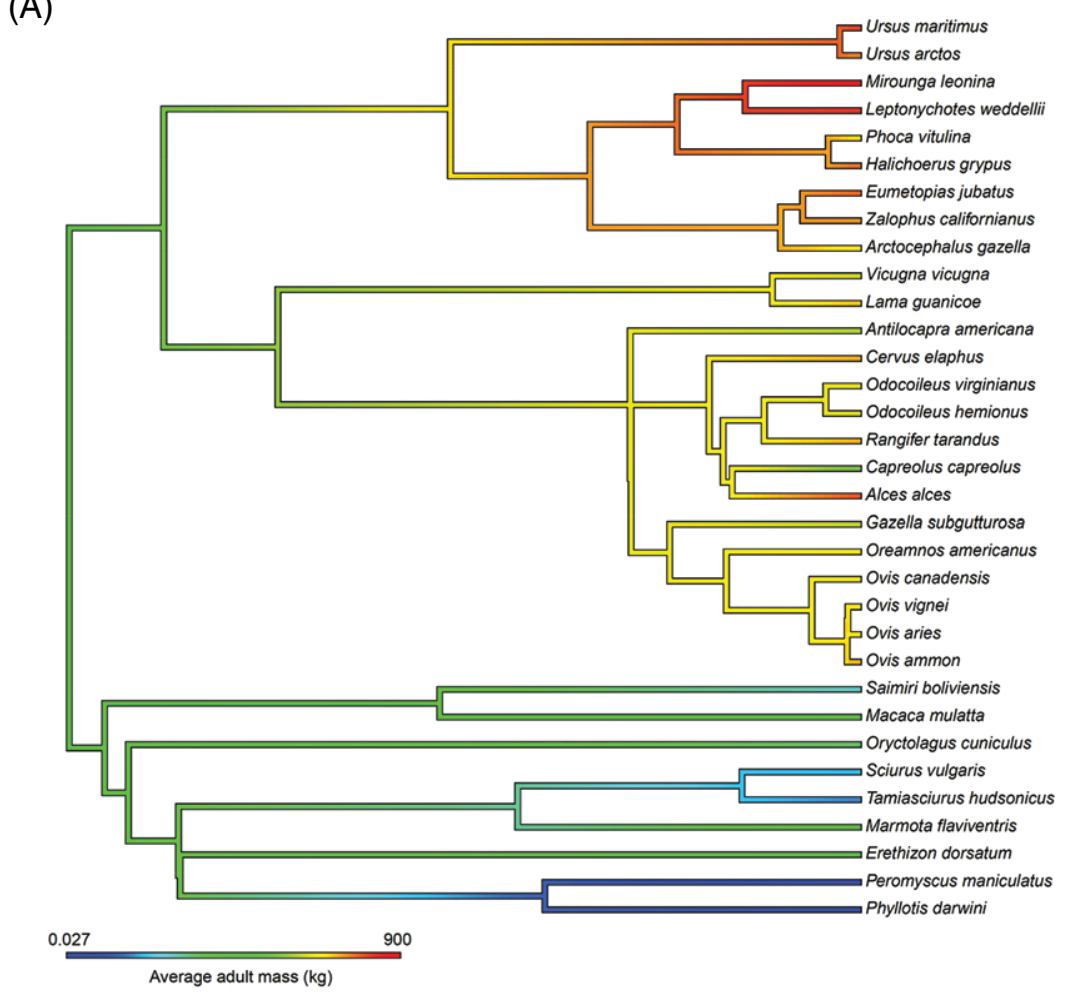

(B)

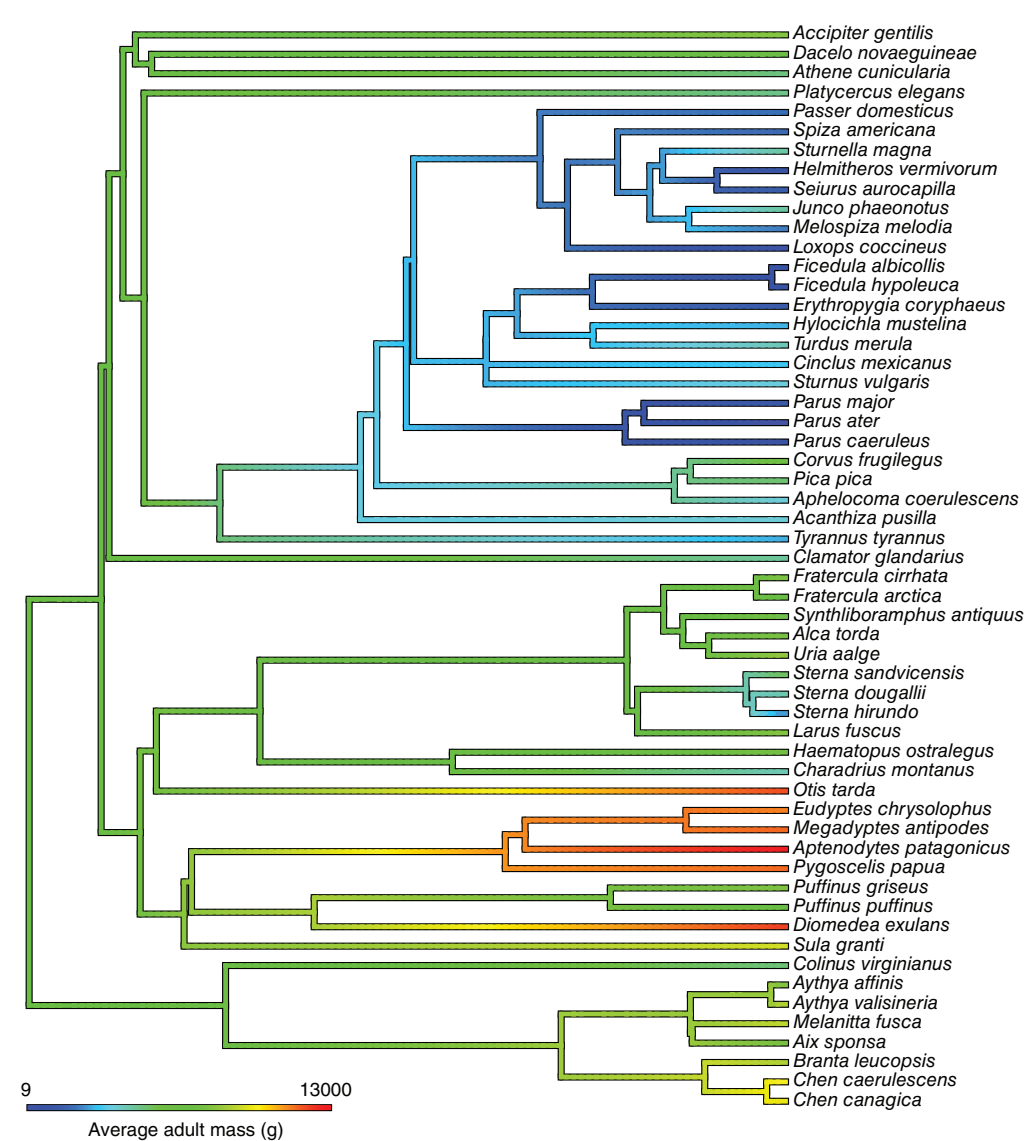

Fig. 2. Phylogenies of (A) mammal (from Bininda-Emonds etal., 2007) and (B) bird (from Jetz et al., 2012) species included in the meta-analyses. The colours indicate the average adult body mass of the species. 
(A)

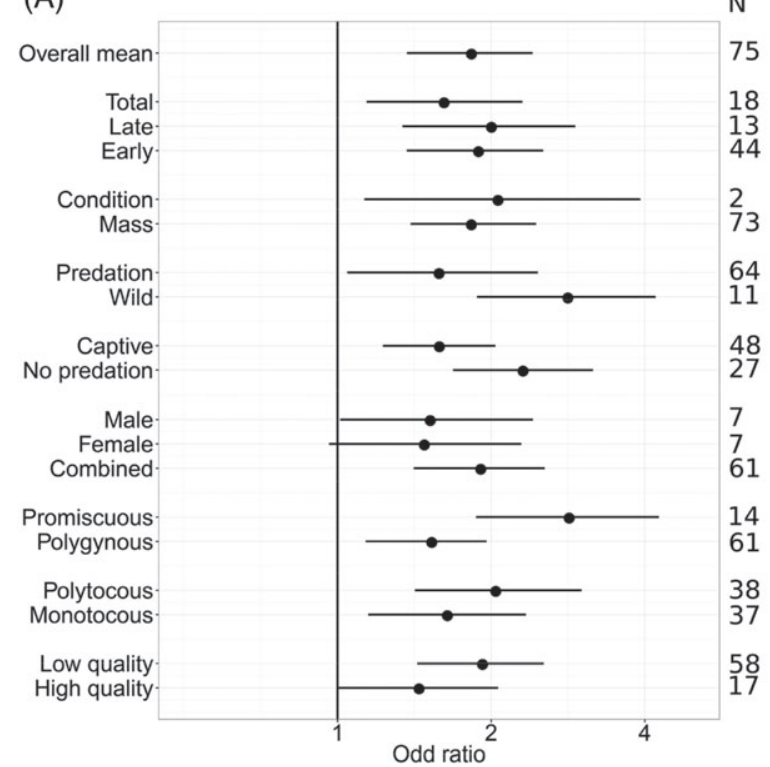

(B)

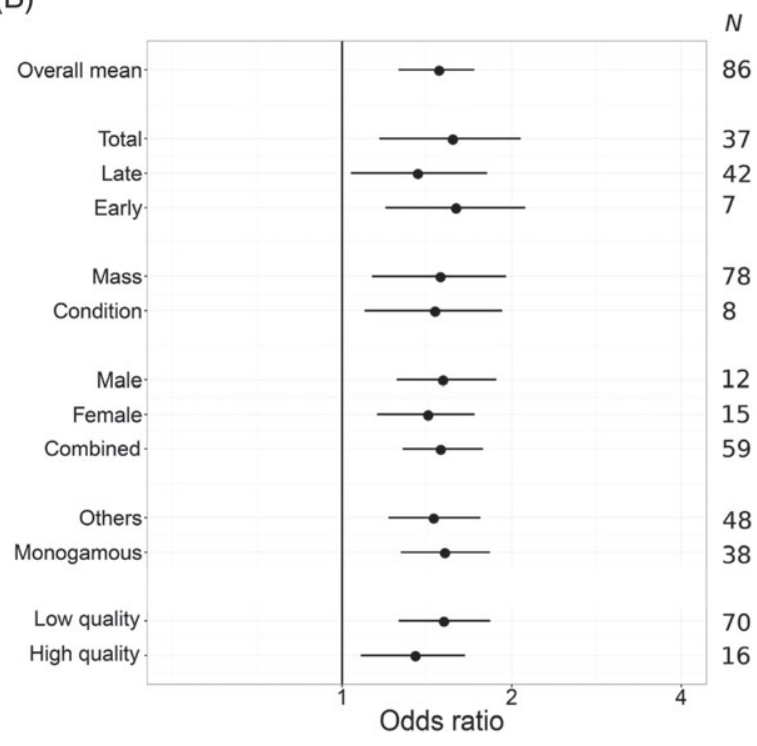

Fig. 3. Meta-analysis means of each moderator (see Section II.4b) for (A) mammals and (B) birds for the relationship between offspring mass and offspring survival. Meta-analysis overall means are also provided. All means are presented with their $95 \%$ highest posterior density intervals and sample size is provided $(\mathcal{N})$.

Table $4 . I^{2}$ value associated with random effect of phylogeny, species, population, and author included when modelling the relationship between offspring mass and offspring survival in mammals and birds. For each value, the lower and upper high posterior density intervals (HPDI) limits of the credibility interval are reported

\begin{tabular}{lccc}
\hline \hline Mammals & Mean & Lower HPDI & Upper HPDI \\
\hline$I^{2}$ Phylogeny & 18.64 & 0.44 & 47.44 \\
$I^{2}$ Species & 18.43 & 0.73 & 44.92 \\
$I^{2}$ Population & 10.29 & 0.54 & 30.66 \\
$I^{2}$ Author & 39.74 & 4.02 & 72.24 \\
$I^{2}$ Residuals & 10.95 & 0.47 & 34.11 \\
\hline \hline Birds & Mean & Lower HPDI & Upper HPDI \\
\hline$I^{2}$ Phylogeny & 26.11 & 2.97 & 54.45 \\
$I^{2}$ Species & 20.26 & 2.24 & 46.39 \\
$I^{2}$ Population & 16.39 & 1.92 & 40.00 \\
$I^{2}$ Author & 26.40 & 2.98 & 56.16 \\
$I^{2}$ Residuals & 10.13 & 1.63 & 24.08 \\
\hline \hline
\end{tabular}

$I^{2}$ near to $25 \%$ for each effect (Table 4). In birds, results were similar with all $I^{2}$ near $25 \%$, which indicates that each random effect included in our meta-analysis accounted for an equal and weak part of the heterogeneity among studies (Table 4). The credibility intervals were large for all the values, preventing us from relying on the exact $I^{2}$ value.

\section{(c) Assessing the effects of moderators on the strength of the offspring mass-survival relationship}

The age at which mass was measured, the type of mass measurement, data quality and sex did not have any detectable effect on the relationship between offspring mass and survival in either birds or mammals (Fig. 3, Table 5). Clutch/litter size did not influence the slope of the relationship either in birds [linear regression slope $=-0.010$, HPDI $=(-0.040 ; 0.021)]$ or in mammals (Table 5). The meta-analysis mean was higher in captive than in wild mammals, and in mammal populations with no predation than in populations subjected to predation. We did not detect any influence of the mating system in birds but promiscuous mammals had a higher meta-analysis mean than polygynous ones, this difference being statistically significant (Table 5). The mating system was not independent from environmental conditions, since $97 \%$ of studies on polygynous species lived in the wild while $64 \%$ of studies on promiscuous species lived in captivity. This prevented us from reaching a firm conclusion on whether mating system influences the offspring mass-survival relationship, because polygynous species in this data set were virtually all from the wild, and our analysis indicated that living in the wild weakens the relationship between offspring mass and survival (see Section IV). Including an interaction between mating system and sex did not reveal any detectable effect either in mammals [ mean $_{\text {malevsfemale promiscuous }}=0.276$, $\mathrm{HPDI}=(-0.394 ; 0.973) ;$ mean $_{\text {male }}$ s female polygynous $=-0.142$, HPDI $=(-0.535 ; \quad 0.222)] \quad$ or $\quad$ in birds $\left[\right.$ mean $_{\text {male vs female monogamous }}=0.013, \quad$ HPDI $=(-0.232$; 0.251); $\quad$ mean $_{\text {male }}$ s female othermating $=-0.100$, HPDI $=(-0.264 ; 0.086)]$. Likewise, we did not find any detectable interaction between litter size and mating system in mammals $\left[\right.$ mean $_{\text {monotocous } v \text { polytocous promiscuous }}=0.181$, $\mathrm{HPDI}=(-0.735 ; 1.067) ;$ mean $_{\text {monotocous vs polytocous polygynous }}$ $=0.085, \mathrm{HPDI}=(-0.307 ; 0.454)]$. 
Table 5. Difference between the log-transformed mean of each moderator for the relationship between offspring mass and offspring survival in mammals and birds with their 95\% high posterior density intervals (HPDI). Values in bold are statistically significant

\begin{tabular}{lccc}
\hline \hline Mammals & Mean difference & Lower HPDI & Upper HPDI \\
\hline Early versus late & 0.058 & -0.341 & 0.439 \\
Early versus total & -0.155 & -0.502 & 0.200 \\
Mass versus condition & 0.120 & -0.480 & 0.765 \\
Gaptive versus wild & $-\mathbf{0 . 5 8 2}$ & $-\mathbf{0 . 1 3 3}$ \\
No predation versus predation & $-\mathbf{0 . 3 7 8}$ & $-\mathbf{0 . 1 2 3}$ \\
Combined sex versus female & -0.255 & $-\mathbf{0 . 6 2 9}$ & 0.186 \\
Combined sex versus male & -0.228 & -0.682 & 0.238 \\
Polygynous versus promiscuous & $\mathbf{0 . 6 2 0}$ & $\mathbf{0 . 6 3 2}$ & $\mathbf{0 2 7}$ \\
Monotocous versus polytocous & 0.218 & -0.144 & 0.608 \\
High quality versus low quality & 0.292 & -0.103 & 0.645 \\
\hline \hline Birds & Mean difference & High HPDI \\
\hline Early versus late & -0.013 & -0.311 & 0.265 \\
Early versus total & -0.156 & -0.427 & 0.128 \\
Condition versus mass & 0.022 & -0.256 & 0.291 \\
Both sex versus female & -0.051 & -0.257 & 0.139 \\
Both sex versus male & 0.010 & -0.177 & 0.229 \\
Monogamous versus other mating & -0.046 & -0.229 & 0.146 \\
High quality versus low quality & 0.116 & 0.306 \\
\hline \hline
\end{tabular}

\section{(d) Publication bias}

The intercept of the Egger regression was statistically different from zero in mammals [intercept $=0.077$, $\mathrm{HPDI}=(0.004,0.152)]$. The publication bias diagram was not symmetrical (Fig. 4A), indicating that a publication bias towards positive effects was likely. The trim and fill method indicated a lack of 18 studies on the left side of the funnel plot. The meta-analytic mean should thus be adjusted by -0.062 , which results in a value of 1.71 . In birds the intercept of the Egger regression also differed from 0 on statistical grounds $[$ intercept $=0.156$, HPDI $=(0.065 ; 0.246)]($ Fig. 4B $)$. The trim and fill method indicated a lack of 15 studies on the left side of the funnel plot. The meta-analytic mean should thus be adjusted by -0.027 , which results in a value of 1.44 . Therefore, the slight publication bias we detected did not influence our conclusions.

\section{(2) Relationship between maternal mass and offspring mass}

(a) Data set

For this meta-analysis, we extracted 96 effect sizes from 60 published papers. We collected effect sizes for 38 different mammalian species with Carnivora (12 species), Rodentia (11 species) and Artiodactyla (10 species) as the most represented mammalian orders (Fig. 5; Table 3). This meta-analysis was performed in mammals only (see Section II.1).

\section{(b) Results from the general meta-analysis}

A positive relationship occurred between offspring and maternal mass (mean meta-analysis $=0.408$, which is equivalent to a Pearson correlation coefficient of 0.387 ).
This effect was statistically significant because the highest posterior density interval did not overlap $0[\mathrm{HPDI}=(0.223$; 0.580)] (Fig. 6).

The heterogeneity analysis showed that all the random effects included in our meta-analysis contributed equally but weakly to the overall heterogeneity across studies, with an $I^{2}$ less than 25\% (Table 6).

\section{(c) Assessing the effects of moderators on the strength of the maternal mass-offspring mass relationship}

The age at which offspring mass was measured, the type of mass measurement, sex, whether animals were captive or not, litter size, data quality, and mating system did not have any detectable effect on the magnitude of the relationship (Fig. 6; Table 7). Likewise, we did not detect any effect of interactions both between mating system and sex $\left[\right.$ mean $_{\text {male vs female promiscuous }}=-0.064$, $\mathrm{HPDI}=(-0.343 ; 0.247) ;$ mean $_{\text {male }} v$ female polygynous $=0.016$, $\mathrm{HPDI}=(-0.010 ; 0.148)]$ and between litter size and mating system $\left[\mathrm{mean}_{\text {monotocous us polytocous promiscuous }}=-0.050\right.$, $\mathrm{HPDI}=(-0.596 ; 0.523) ;$ mean $_{\text {monotocous } v \text { polytocous polygynous }}$ $=0.044$, HPDI $=(-0.151 ; 0.248)]$.

\section{(d) Publication bias}

The intercept of the Egger regression was almost statistically different from zero [intercept $=0.037, \mathrm{HPDI}=(-0.001$; $0.075)]$. A direct inspection of the diagram suggests that some studies might be lacking on the left side since the funnel plot is not symmetrical (Fig. 7). This indicates that a small publication bias might exist. However, the results of the Egger regression indicate that our results are robust to such a small bias. 
(A)

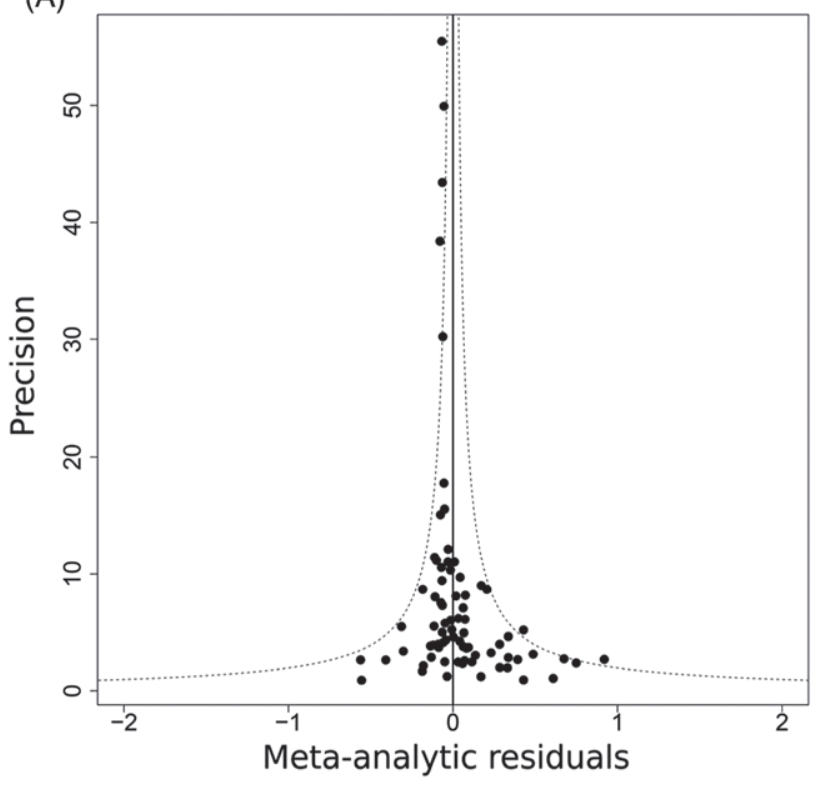

(B)

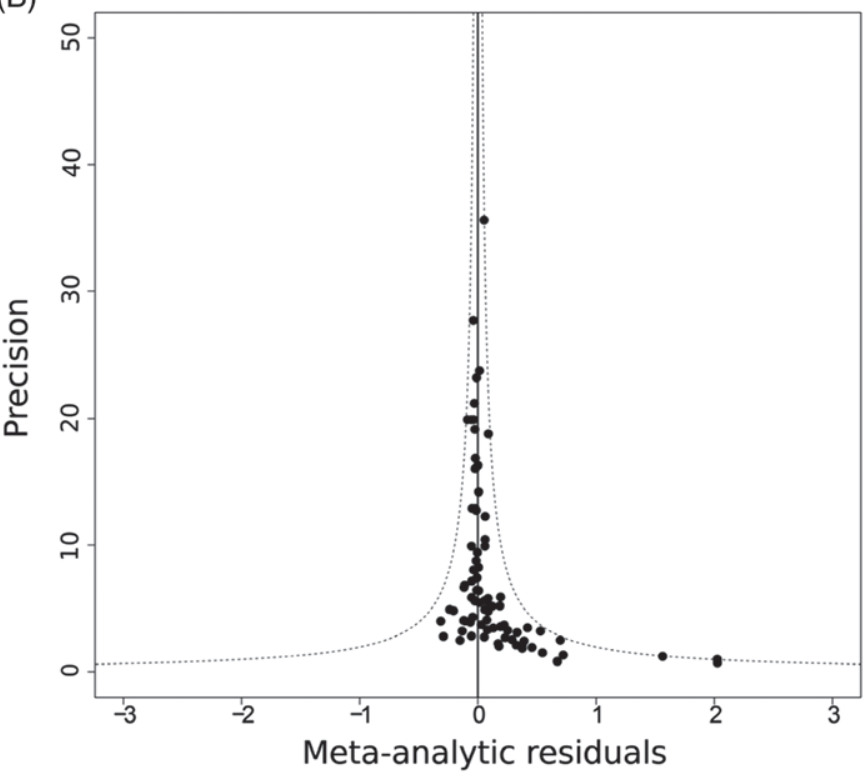

Fig. 4. Funnel plots of the different effect sizes in (A) mammals and (B) birds for the relationship between offspring mass and survival. The precision is plotted as a function of the meta-analysis residuals, as recommended by Nakagawa \& Santos (2012). The vertical solid line corresponds to 0 .

\section{DISGUSSION}

We assessed the sign and the magnitude of the relationships between offspring mass and offspring survival in mammals and birds and between maternal and offspring mass in mammals. The meta-analyses we performed provided strong support for positive relationships in all cases.

In mammals, on average, when offspring mass increases by 1 standard deviation of the offspring body mass distribution in the population, the odds of offspring survival increase by $71 \%$. We also highlighted the existence of a positive relationship between offspring mass and survival in birds. On average, when offspring mass increases by one standard deviation of the early mass distribution the odds of offspring survival increase by $44 \%$. Overall, these positive relationships support our expectation that offspring mass is a reliable proxy of individual survival in birds and mammals (e.g. Hamel etal., 2009). The magnitude of the relationship was slightly weaker in birds. This difference might be due to the fact that birds and mammals are not subject to the same constraints. As $92 \%$ of our effect sizes were measured on post-fledging survival, flight constraints are likely involved. The advantages of a greater body mass in birds might be not so strong because a high body mass increases the wing loading (Chandler \& Mulvihill, 1992) and affects birds in terms of flying performance (Norberg, 1995). There is an extensive literature about the cost of being too fat, especially when individuals need high flight performance to escape predators (e.g. Gosler, Greenwood \& Perrins, 1995; Bonter et al., 2013; Rogers, 2015). In birds, there is clearly a trade-off between the advantage of being fat to avoid starvation and its costs in terms of predation. Alternatively, a methodological issue might account for the weaker influence of mass on juvenile survival in birds compared to mammals. In bird studies, it is especially difficult to distinguish between death and emigration from the study site (Lebreton etal., 1992; Lebreton, Pradel \& Clobert, 1993; Gilroy et al., 2012). When the probability of emigration increases with body mass, the relationship between resighting rate (often used as a proxy of survival) and mass is weaker than the relationship between true survival and mass (Stoleson \& Beissinger, 1997; Barbraud, Johnson \& Bertault, 2003).

From the heterogeneity analysis, we found weak effects of phylogeny, of species independent of phylogeny, and of population. As neither among-species nor among-population differences accounted for a substantial proportion of the variation observed in the strength of the relationship between offspring survival and body mass, we can generalize our results to all mammals and birds. The absence of any detectable random effect to explain part of the heterogeneity highlights the importance of environmental variation on shaping these relationships. Juveniles from the same species can die from different causes and, even within the same population, juveniles born in different cohorts do not face the same environment (e.g. Keech etal., 2011; Garratt et al., 2015). In both birds and mammals, data quality did not influence our finding because we did not detect any difference between the mean of low-quality data and that of high-quality data. While a publication bias was detected in birds and to a lesser extent in mammals, it only involved a negligible decrease of the overall effect size, which left our conclusions unchanged.

To find potential major drivers explaining the variation in slopes reported in the literature for the offspring 


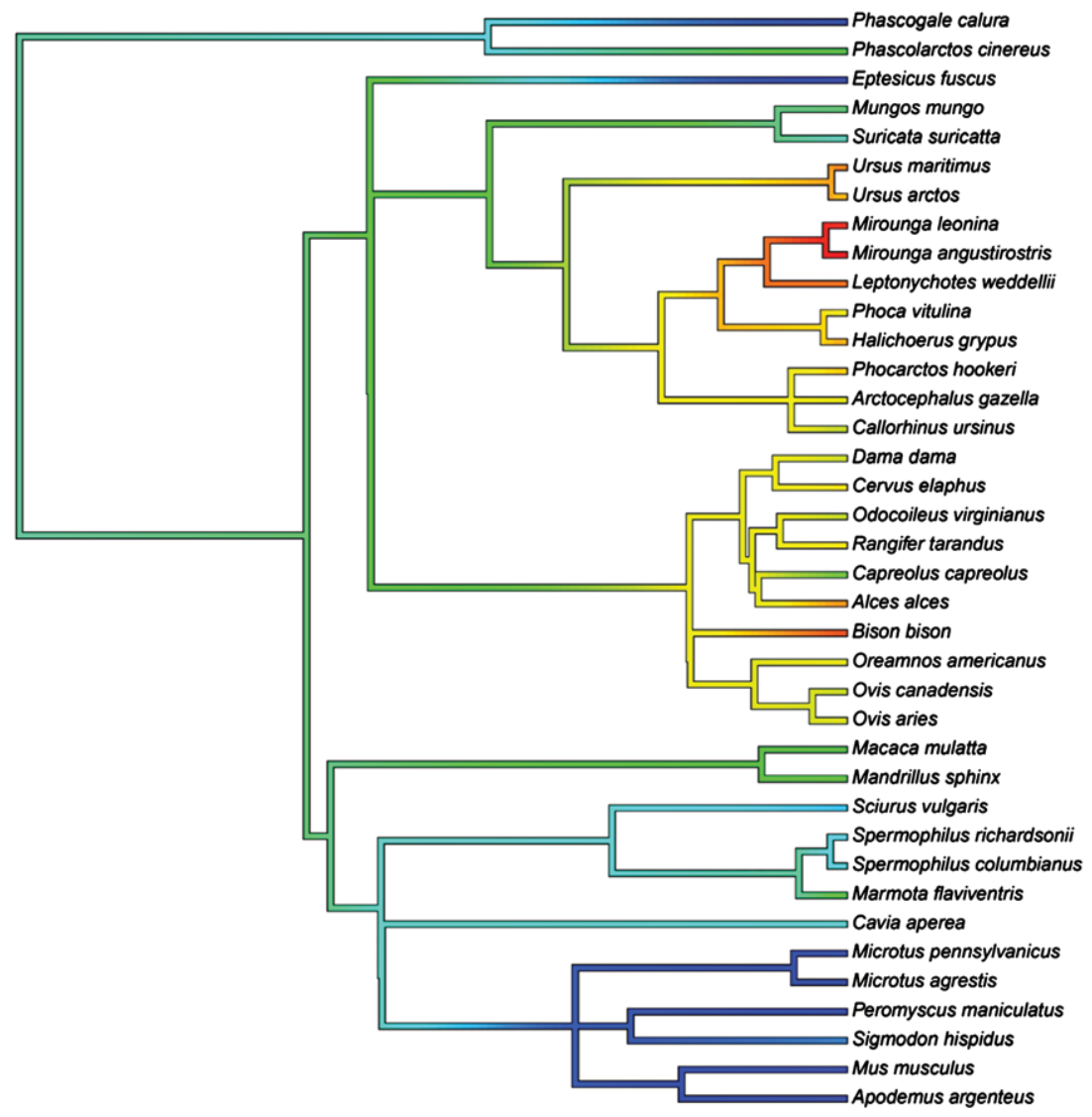

\section{$0.02 \quad 1600$}

Fig. 5. Phylogeny of mammal species included in the analysis of the relationship between offspring mass and maternal mass (from Bininda-Emonds et al., 2007). The colours indicate the average adult body mass of the species.

mass-survival relationship, we tested potential effects of the timing of the measurement. We examined three periods, including the period with high parental care from birth to weaning/fledging, the period of juvenile independence from weaning/fledging to adult stage, and the overall juvenile survival from birth to adult stage. A general objective behind this analysis was to assess in which period juvenile survival is most dependent on body mass. We did not identify a critical period likely because such effects could be masked by dominant mortality causes like predation, which is often less body-mass dependent than other causes of mortality such as starvation (Monteith etal., 2014). A negative effect of predation on the strength of the offspring mass-survival relationship is confirmed by our findings in mammals that offspring survival in populations subjected to predation is less closely associated with body mass. However, the effective predation rate might strongly influence the strength of condition-dependence, which is expected to peak at some intermediate value of predation rate. Unfortunately, predation rates for the mammalian populations considered in our meta-analysis were not provided and it was thus impossible to assess accurately how predation affects the mass-survival relationship. It is also noteworthy that absolute body mass as analysed here might not reflect condition-dependent mortality through predation. Indeed, if we assume the existence of a limited mass range over which predators are able to prey upon juveniles, all juveniles in a population will be susceptible to predation initially, but the duration of the vulnerability period will be much lower for fast-growing juveniles. In such cases, which encompass most ungulates (Byers, 1997), condition-dependent mortality is weak when using absolute body mass but could be much stronger when using individual growth rate instead of mass. In birds, several studies have reported that the critical period in terms of survival occurs just after fledging because the newly independent juveniles have little experience in foraging and so have to rely on their body reserves, which could be expected to strengthen the relationship between mass and survival (e.g. Sullivan, 1989; Stienen \& Brenninkmeijer, 2002). However, fledging in birds also corresponds to a period when other causes of mortality occur, such as predation, likely explaining why late survival is not strongly associated with condition (Davies \& Restani, 2006). The relationship between offspring survival and offspring body mass is driven 


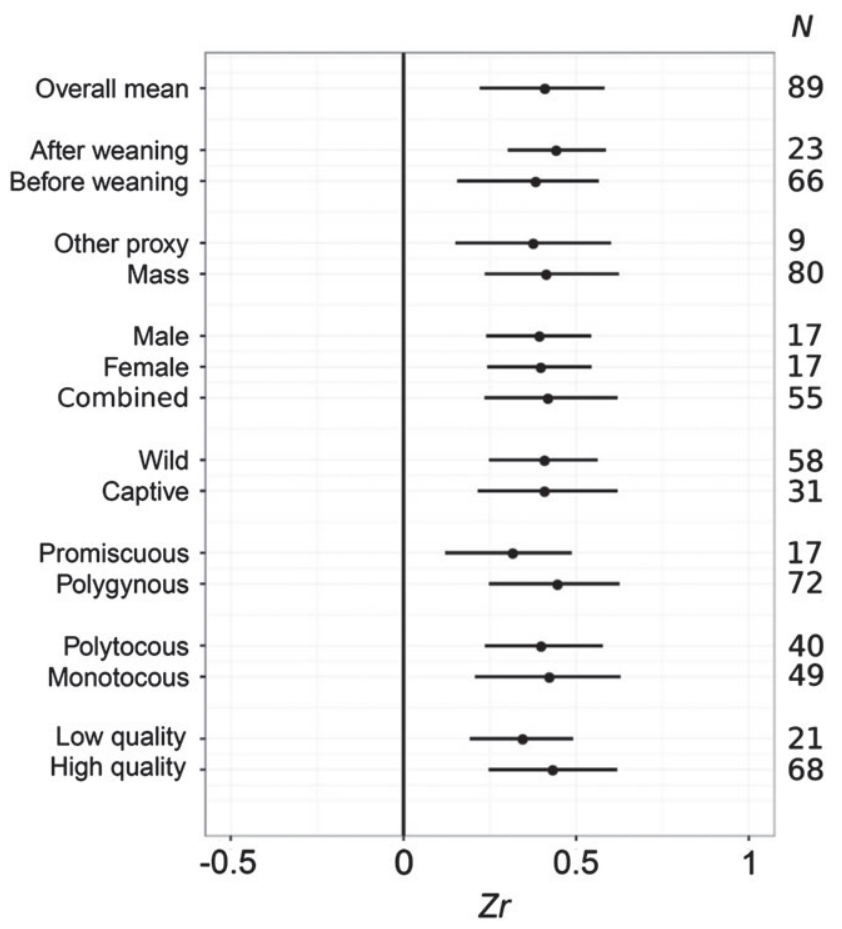

Fig. 6. Meta-analysis means of each moderator (see Section II. $4 b$ ) in mammals for the relationship between offspring mass and maternal mass. Meta-analysis overall means are also provided. All means are presented with their 95\% highest posterior density intervals and sample size is provided $(\mathcal{N})$.

Table 6. $I^{2}$ value associated with random effect of phylogeny, species, population, and author when modelling the relationship between offspring mass and maternal mass in mammals. For each value, the lower and upper high posterior density interval (HPDI) limits of the credibility interval are reported

\begin{tabular}{lccc}
\hline \hline Mammals & Mean & Lower HPDI & High HPDI \\
\hline$I^{2}$ Phylogeny & 24.15 & 3.39 & 47.72 \\
$I^{2}$ Species & 16.10 & 2.92 & 34.79 \\
$I^{2}$ Population & 14.19 & 2.98 & 31.28 \\
$I^{2}$ Author & 19.58 & 3.20 & 39.71 \\
$I^{2}$ Residuals & 16.73 & 3.08 & 34.21 \\
\hline \hline
\end{tabular}

by two parameters: the proportion of total mortality that is condition-independent or weakly condition-dependent and the strength of the relationship for each type of condition-dependent mortality. Condition-dependent mortality is mainly caused by starvation in relation to the depletion of body reserves of the juveniles (Williams \& Croxall, 1991). As we compiled studies over a large range of environmental conditions and mortality causes, the absence of any influence of the juvenile period studied is not so surprising.

Surprisingly, survival of captive mammals was more dependent on body mass than that of wild mammals. Wild animals have to face a much larger range of mortality factors, such as predation, which is often a major cause of offspring mortality (e.g. Linnell, Aanes \& Andersen, 1995 in ungulates). Although accurate estimates of predation rates in the wild are generally lacking, it seems likely that predation, which is likely to be the highest during a limited time window of the juvenile stage, is only weakly related to absolute juvenile body mass. On the contrary, juveniles in captive populations are not subjected to predation and mostly die from infectious diseases or starvation, which can occur over the entire juvenile stage and are highly associated with absolute body mass (Yapi, Yapi, Boylan \& Robinson, 1990; Mandal etal., 2007). To assess the offspring mass-survival relationship in multiple case studies, different measures of mass were included. The most commonly used metric other than mass was body condition (i.e. mass corrected for size; Schulte-Hostedde et al., 2005). Such heterogeneity in mass measurements could have led to an increase in variance across studies. However, a relatively low number of studies based on body condition were included in our analyses (two out of 75 for mammals and eight out of 86 for birds). Using other phenotypic traits to assess condition, such as growth rate, would improve our understanding of condition-dependent juvenile mortality.

We did not find any effect of sex on the magnitude of the relationship in mammals or birds. In particular, we did not find any evidence for disproportionately larger survival or mass advantage of increasing offspring mass in males than in females during early stages of life even when we accounted for the potential confounding effect of mating systems. However, these results do not necessarily contradict the Trivers-Willard Hypothesis (Trivers \& Willard, 1973) because we only looked at the early stages of life, whereas, as recently demonstrated, sex-specific reproductive value across the whole life course has to be considered to predict reliably a selective pressure for sex-biased allocation, even in the most sexually dimorphic and polygynous species (Schindler etal., 2015). Among the species-specific reproductive life-history traits, we considered only the mating system in mammals, which had a detectable influence on the offspring mass advantage. Offspring survival was more strongly mass-dependent in promiscuous species than in polygynous species. However, as the mating system had a confounding effect with environmental conditions, we cannot firmly conclude which of these factors generated the observed relationship. Furthermore, we did not find a higher effect of offspring body mass in polytocous and promiscuous mammal species for which we expected high sibling competition due to the existence of multi-paternity within litters.

In mammals, maternal mass was positively correlated to offspring mass with a mean correlation coefficient of 0.387 , which corresponds to a moderate effect (sensu Cohen, 1988). This finding matches the expectation that heavier mothers in a given population allocate more to their offspring than lighter ones, by allowing offspring to reach higher body mass and thereby higher survival. Interestingly, this finding supports recent results reported by Lim etal. (2014) who found a correlation coefficient of 0.414 between maternal size and offspring size for a wider set of taxonomic groups. 
Table 7. Difference between the mean of each moderator for the relationship between offspring mass and maternal mass in mammals with their 95\% high posterior density interval (HPDI)

\begin{tabular}{lccc}
\hline \hline Mammals & Mean difference & Lower HPDI & Upper HPDI \\
\hline Before versus after weaning & 0.060 & -0.078 & 0.203 \\
Mass versus other proxy & -0.038 & -0.259 & 0.188 \\
Both sex versus female & -0.021 & -0.173 & 0.125 \\
Both sex versus male & -0.025 & -0.176 & 0.125 \\
Captive versus wild & -0.001 & -0.156 & 0.154 \\
Polygynous versus promiscuous & -0.123 & -0.322 & 0.041 \\
Monotocous versus polytocous & -0.025 & -0.185 & 0.154 \\
High quality versus low quality & -0.087 & -0.237 & 0.059 \\
\hline
\end{tabular}

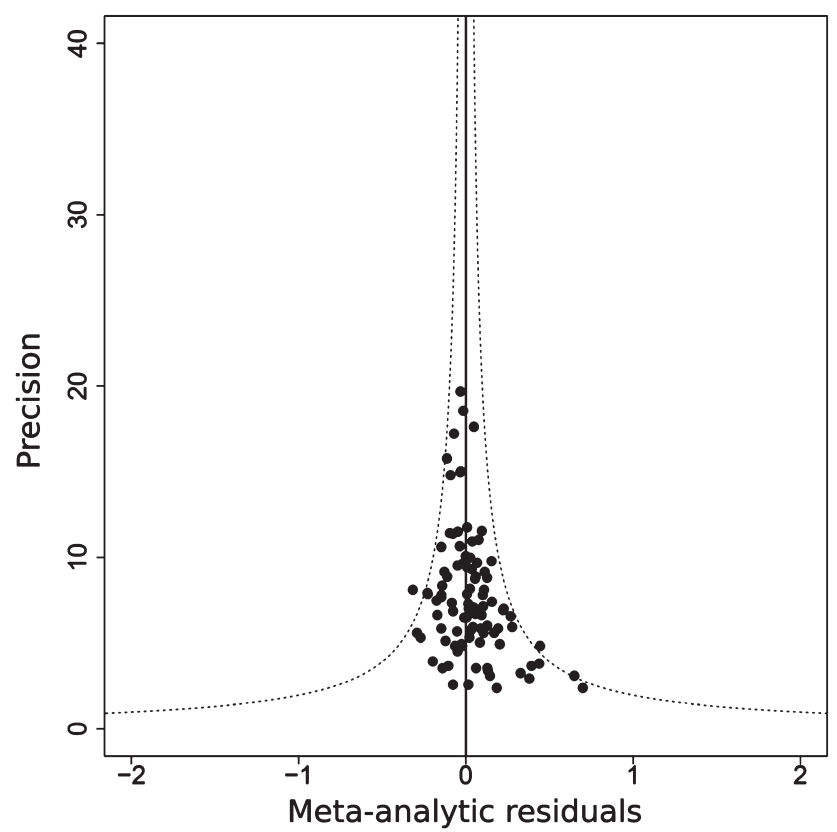

Fig. 7. Funnel plots of the different effect sizes in mammals for the relationship between offspring mass and maternal mass. The precision is plotted as a function of the meta-analysis residuals, as recommended by Nakagawa \& Santos (2012). The vertical solid line corresponds to 0 .

The generally strong size-mass relationship explains the consistency of results across studies (e.g. Dahle, Zedrosser \& Swenson, 2006).

Both the species and the population random effects only accounted for a weak proportion of observed heterogeneity in our meta-analysis, which indicates that the positive effect we highlighted is consistent across mammalian species. As we included a large diversity of mammals, we can safely generalize our findings to the entire class of mammals. The type of data used did not influence the results and the publication bias we detected had only a very weak effect on the final result. We were not able to perform this analysis for birds because of insufficient data. In birds more effort has been allocated to studying the relationship between maternal mass and egg mass, which is likely to be positive (Wiggins, 1990; Budden \& Beissinger, 2005). Egg mass also relates to neonatal mass in birds (Krist, 2011), which leads us to expect that the relationship we identified in mammals also holds in birds. The few studies that assessed the maternal mass-offspring mass relationship in birds supported the existence of a positive relationship between maternal mass and offspring mass (Blums, Clark \& Mednis, 2002; Parker, 2002; Newbrey \& Reed, 2009).

We checked whether the timing of the offspring measurement could impact the magnitude of the mother-offspring mass relationship. Measuring offspring before weaning or after weaning led to similar results. Maternal mass thus provides a reliable predictor of both offspring birth mass and weaning mass in mammals. This result is not surprising because weaning mass is highly related to birth mass in mammals, with weaning mass being about four times the birth mass in pinnipeds, primates, and ungulates (Lee et al., 1991). As in the analysis of the offspring mass-survival relationship, the use of different types of measurement did not have any impact on this meta-analysis. Likewise, wild and captive mammalian females allocate to their offspring with the same intensity at a given size. This is quite surprising when considering that body mass is more closely related to offspring survival in captive than in wild populations. However, we expect that females should increase their offspring body mass relative to their own mass only if an increase of the offspring body mass can give a sufficient increase in offspring survival compared to lighter ones. In captivity offspring body mass is more closely related to survival than in the wild but average offspring survival is typically higher in captivity than in the wild (Littleton, 2005). Because offspring survival is already high in captivity, any increase in offspring body mass might not provide additional survival benefits.

The absence of any sex difference on the maternaloffspring mass relationship was an unexpected result, which indicates that mothers allocate the same relative amount of energy to male and female offspring irrespective of their body mass. Similar results were found in birds with no sex-biased allocation to egg size (Rutkowska, Dubiec \& Nakagawa, 2014). In polygynous species the disproportionate mass or size advantage of offspring males is expected to be higher than in promiscuous species (Clutton-Brock, 1991) but we did not find any interaction between offspring sex 
and mating system. However, a similar correlation coefficient does not imply that there is no differential allocation between sexes. For a given mass, mothers can produce heavier male than female offspring. We did not detect any difference between the correlation coefficients but the intercept of the relationship was generally higher in males, indicating that mothers allocate more to male than to female offspring (e.g. Foster \& Taggart, 2008). Furthermore, the mother can also allocate more toward males by biasing offspring sex ratio instead of increasing male mass, explaining why in some cases maternal mass can be related to offspring sex ratio (Arnbom, Fedak \& Boyd, 1997). In addition, as recently pointed out by Schindler etal. (2015), the full sex-specific reproductive value has to be considered before stating that there are adaptive sex differences in maternal care. In 11 of our 17 studies that tested such differences, offspring mass was measured at birth, meaning that all the maternal allocation after birth was not accounted for.

Interestingly we did not find any difference in female allocation to offspring mass between monotocous and polytocous mammals. Mammals that produce multiple offspring can modify maternal allocation via two pathways: the offspring mass or the offspring number. We thus expected females of polytocous mammals to allocate less to offspring mass than females of monotocous species. However, in most cases, the expected offspring size-number trade-off does not show up among females within a population and both the mean mass of offspring and litter size increase with maternal mass (reviewed by Lim et al., 2014). The mating system does not seem to impact this relationship. This is not so surprising because the difference between promiscuous and polygynous mating systems is only expected to impact paternal allocation (Adrian etal., 2005). As the degree of paternity certainty is lower in promiscuous species than in polygynous species, promiscuous fathers should allocate less than polygynous fathers (Wright \& Cotton, 1994), whereas such differences are not expected for maternal allocation.

\section{GONGLUSIONS}

(1) Using meta-analyses we provide strong evidence of a positive relationship between offspring mass and offspring survival in birds and mammals. Our main finding shows the importance of considering body mass when analysing variation in early survival. Offspring mass offers a reliable indicator of offspring survival in both birds and mammals. However, the magnitude of the relationship was weaker for birds, likely because of flight constraints.

(2) We did not identify biological drivers that explained the differences we observed in the magnitude of the offspring mass-survival relationship across studies. We propose that this is because the offspring mass-survival relationship is highly dependent on the mortality causes in the focal populations. When most individuals die from weakly condition-dependent factors such as predation, a low magnitude of the relationship is expected, whereas when condition-dependent factors such as starvation mostly cause mortality, a higher magnitude of the relationship is expected.

(3) Offspring body mass, which drives individual differences in survival among offspring, is positively correlated with maternal body mass in mammals. This correlation was not quantitatively tested in birds due to a lack of data. However, from the limited information collected so far, there is support for a positive relationship. Further work, when sufficient data are available, should assess the correlation coefficient in birds for comparison with the coefficient obtained here for mammals. Because offspring survival is less related to offspring mass in birds, we expect a smaller coefficient of correlation in birds than in mammals.

(4) We did not identify any major driver that could explain the observed variability in the relationship between maternal mass and offspring mass. As we found large variation in condition-dependent survival in mammals in relation to variation in environmental conditions, we expected also to find large variation in the relationship between offspring and maternal mass. The link between the two relationships studied here is not clear and is worth further investigation.

\section{AGKNOWLEDGEMENTS}

V. R. is funded by the French ministry of Education and Research. We thank Heiko Rödel, Martin Postma and Géraldine Mabille for generous provision of information about their studies. We are especially grateful to John Byers and an anonymous reviewer for helpful comments.

\section{REFERENCES}

*References marked with an asterisk have been included in the meta-analyses.

*Adams, L. G. (2005). Effects of maternal characteristics and climatic variation on birth masses of Alaskan caribou. Foumal of Mammalogy 86, 506-513.

Adrian, O., Brockmann, I., Hohoff, G. \& Sachser, N. (2005). Paternal behaviour in wild guinea pigs: a comparative study in three closely related species with different social and mating systems. Fournal of Zoology 265, 97-105.

*Anderson, M. G., LindberG, M. S. \& Emery, R. B. (2001). Probability of survival and breeding for juvenile female canvasbacks. Foumal of Wildlife Management 65, 385-397.

*Arnbom, T., Fedak, M. A. \& Boyd, I. L. (1997). Factors affecting maternal expenditure in southern elephant seals during lactation. Ecology 78, 471-483.

*Awan, G. A., Festa-Bianchet, M. \& Gaillard, J. M. (2008). Early survival of Punjab urial. Canadian fournal of Zoology 86, 394-399.

*Barber-Meyer, S. M., Mech, L. D. \& White, P. J. (2008). Elk calf survival and mortality following wolf restoration to Yellowstone National Park. Wildlife Monographs 169, $1-30$.

Barbraud, C., Johnson, A. R. \& Bertault, G. (2003). Phenotypic correlates of post-fledging dispersal in a population of greater flamingos: the importance of body condition. Fournal of Animal Ecology 72, 246-257.

Bennett, K. A., Hughes, J., Stamatas, S., Brand, S., Foster, N. L., Moss, S. E. \& Pomeroy, P. P. (2015). Adiponectin and insulin in gray seals during suckling and fasting: relationship with nutritional state and body mass during nursing in mothers and pups. Physiological and Biochemical Zoology 88, 295-310.

*Bercovitch, F. B., Widdig, A. \& Nürnberg, P. (2000). Maternal investment in rhesus macaques (Macaca mulatta): reproductive costs and consequences of raising sons. Behavioral Ecology and Sociobiology 48, 1-11.

*Berger, J. (2012). Estimation of body-size traits by photogrammetry in large mammals to inform conservation. Conservation Biology 26, 769-777.

Bininda-Emonds, O. R. P., Cardillo, M., Jones, K. E., MacPhee, R. D. E. Beck, R. M. D., Grenyer, R., Price, S. A., Vos, R. A., Gittleman, J. L. \& Purvis, A. (2007). The delayed rise of present-day mammals. Nature 446, 507-512. 
*Birgersson, B. \& Ekvall, K. (1997). Early growth in male and female fallow deer fawns. Behavioral Ecology 8, 493-499.

*Bishop, C. J., Unsworth, J. W. \& Garton, E. O. (2005). Mule deer survival among adjacent populations in southwest Idaho. Journal of Wildlife Management 69, $311-321$.

*Bishop, G. J., White, G. G., Freddy, D. J., Watkins, B. E. \& Stephenson, T. R. (2009). Effect of enhanced nutrition on mule deer population rate of change. Wildlife Monographs 177, 1-28.

*Blaxter, K. L. \& Hamilton, W. J. (1980). Reproduction in farmed red deer. II. Calf growth and mortality. Fournal of Agricultural Science 95, 275-284.

*Blomquist, G. E. \& Williams, L. E. (2013). Quantitative genetics of costly neonatal sexual size dimorphism in squirrel monkeys (Saimiri boliviensis). Foumal of Evolutionary Biology 26, 756-765.

Blums, P., Clark, R. G. \& Mednis, A. (2002). Patterns of reproductive effort and success in birds: path analyses of long-term data from European ducks. Fournal of Animal Ecology 71, 280-295.

*Boltnev, A. I. \& York, A. E. (2001). Maternal investment in northern fur seals (Callorhinus ursinus): interrelationships among mothers' age, size, parturition date, offspring size and sex ratios. Fournal of Zoology 254, 219-228.

*Bolton, M. (1991). Determinants of chick survival in the lesser Black-Backed Gull: relative contributions of egg size and parental quality. Fournal of Animal Ecology 60, 949-960.

*Bonenfant, C., Gaillard, J. M., Loison, A. \& Klein, F. (2003). Sex-ratio variation and reproductive costs in relation to density in a forest-dwelling population of red deer (Cervus elaphus). Behavioral Ecology 14, 862-869.

Bonter, D. N., Zuckerberg, B., Sedgwick, C. W. \& Hochachka, W. M. (2013). Daily foraging patterns in free-living birds: exploring the predation-starvation trade-off. Proceedings of the Royal Society B: Biological Sciences 280, 20123087.

*Booher, C. M. (2008). Effects of calcium availability on reproductive output of big brown bats. Fournal of Zoology 274, 38-43.

*Bowen, W. D., Ellis, S. L., Iverson, S. J. \& Boness, D. J. (2001a). Maternal effects on offspring growth rate and weaning mass in harbour seals. Canadian fournal of Zoology 79, 1088-1101.

*Bowen, W. D., Iverson, S. J., Boness, D. J. \& Oftedal, O. T. (2001b). Foraging effort, food intake and lactation performance depend on maternal mass in a small phocid seal. Functional Ecology 15, 325-334.

*Bowen, W. D., Oftedal, O. T., Boness, D. J. \& Iverson, S. J. (1994). The effect of maternal age and other factors on birth mass in the harbour seal. Canadian fournal of Zoology 72, 8-14.

*Boyd, I. L. \& McCann, T. S. (1989). Pre-natal investment in reproduction by female Antarctic fur seals. (Arctocephalus gazella). Behavioral Ecology and Sociobiology 24, 377-385.

*Braasch, A., Schauroth, C. \& Becker, P. H. (2009). Post-fledging body mass as a determinant of subadult survival in common terns Sterna hirundo. Fournal of Ornithology 150, 401-407.

*Brown, W. P. \& Roth, R. R. (2004). Juvenile survival and recruitment of wood thrushes Hylocichla mustelina in a forest fragment. Fournal of Avian Biology 35, 316-326.

Budden, A. E. \& Beissinger, S. R. (2005). Egg mass in an asynchronously hatching parrot: does variation offset constraints imposed by laying order? Oecologia 144, $318-326$.

Byers, J. A. (1997). American Pronghorn. Social Adaptations and the Ghosts of Predators Past. The University of Chicago Press, Chicago \& London.

Cam, E., Link, W. A., Cooch, E. G., Monnat, J. Y. \& Danchin, E. (2002). Individual covariation in life-history traits: seeing the trees despite the forest. The American Naturalist 159, 96-105.

*Campbell, M. T. \& Slade, N. A. (1995). The effect of maternal mass on litter size and offspring survival in the hispid cotton rat (Sigmodon hispidus). Canadian Fournal of Zoology 73, 133-140.

*Carstensen, M., Delgiudice, G. D., Sampson, B. A. \& Kuehn, D. W. (2009). Survival, birth characteristics, and cause-specific mortality of white-tailed deer neonates. Fournal of Wildlife Management 73, 175-183.

* Casellas, J., Caja, G., Such, X. \& Piedrafita, J. (2007). Survival analysis from birth to slaughter of Ripollesa lambs under semi-intensive management. Fournal of Animal Science 85, 512-517.

Caswell, H. (2001). Matrix Population Models. John Wiley \& Sons, Ltd, Chichester.

Chandler, G. R. \& Mulvihill, R. S. (1992). Effects of age, sex, and fat level on wing loading in dark-eyed juncos. The Auk 109, 235-241.

Charnov, E. L. \& Ernest, S. K. M. (2006). The offspring-size/clutch-size trade-off in mammals. The American Naturalist 167, 578-582.

*Chilvers, B. L., Robertson, B. C., Wilkinson, I. S. \& Duignan, P. J. (2007). Growth and survival of New Zealand sea lions, Phocarctos hookeri: birth to 3 months. Polar Biology 30, 459-469.

*Cleasby, I. R., Nakagawa, S., Gillespie, D. O. S. \& Burke, T. (2010). The influence of sex and body size on nestling survival and recruitment in the house sparrow. Biological Journal of the Linnean Society 101, 680-688.

Clutton-Brock, T. H. (1991). The Evolution of Parental Care. Princeton University Press, Princeton.
Clutton-Brock, T. H., Albon, S. D. \& Guinness, F. E. (1985). Parental investment and sex differences in juvenile mortality in birds and mammals. Nature 313, 131-133. *Clutton-Brock, T. H., Albon, S. D. \& Guinness, F. E. (1986). Great expectations: dominance, breeding success and offspring sex ratios in red deer. Animal Behaviour 34, 460-471.

Clutton-Brock, T. H. \& Sheldon, B. C. (2010). Individuals and populations: the role of long-term, individual-based studies of animals in ecology and evolutionary biology. Trends in Ecology \& Evolution 25, 562-573.

*Clutton-Brock, T. H., Stevenson, I. R., Marrow, P., MacColl, A. D., Houston, A. I. \& McNamara, J. M. (1996). Population fluctuations, reproductive costs and life-history tactics in female Soay sheep. Fournal of Animal Ecology 65, $675-689$.

Cohen, J. (1988). Statistical Power Analysis for the Behavioral Studies. Academic Press, New York.

*Coltman, D. W., Bowen, W. D. \& Wright, J. M. (1998). Birth weight and neonatal survival of harbour seal pups are positively correlated with genetic variation measured by microsatellites. Proceedings of the Royal Society B: Biological Sciences 265, 803-809.

*Cooch, E. G. (2002). Fledging size and survival in snow geese: timing is everything (or is it?). Fournal of Applied Statistics 29, 143-162.

*Côté, S. D. \& Festa-Bianchet, M. (2001). Birthdate, mass and survival in mountain goat kids: effects of maternal characteristics and forage quality. Oecologia $127,230-238$.

*Crocker, D. E., Williams, J. D., Costa, D. P. \& Le Boeuf, B. J. (2001). Maternal traits and reproductive effort in northern elaphant seals. Ecology 82, 3541-3555.

*Dahle, B., Zedrosser, A. \& Swenson, J. E. (2006). Correlates with body size and mass in yearling brown bears (Ursus arctos). Fournal of Zoology 269, 273-283.

*Davies, J. M. \& Restani, M. (2006). Survival and movements of juvenile burrowing owls during the postfledging period. The Condor 108, 282-291.

*Davis, J. B., Cox, R. R. J., Kaminski, R. M. \& Leopold, B. D. (2007). Survival of wood duck ducklings and broods in Mississippi and Alabama. Journal of Wildlife Management 71, 507-517.

*Derocher, A. E. \& Stirling, I. (1994). Age-specific reproductive performance of female polar bears (Ursus maritimus). Fournal of Zoology 234, 527-536.

*Derocher, A. \& Stirling, I. (1996). Aspects of survival in juvenile polar bears. Canadian foumal of Zoology 74, 1246-1252.

*Dinsmore, S. J., White, G. C. \& Knopf, F. L. (2003). Annual survival and population estimates of mountain plovers in southern Phillps County, Montana. Ecological Applications 13, 1013-1026.

*Ditchkoff, S. S., Sams, M. G., Lochmiller, R. L. \& Leslie, D. M. Jr. (2001). Utility of tumor necrosis factor- $\alpha$ and interleukin-6as predictor of neonatal mortality in white-tailed deer. Fournal of Mammalogy 82, 239-245.

*Dobson, F. S. \& Michener, G. R. (1995). Maternal traits and reproduction in Richardson's ground squirrels. Ecology 76, 851-862.

*Dobson, F. S. \& Myers, P. (1989). The seasonal decline in the litter size of meadow voles. Fournal of Mammalogy 70, 142-152.

*Dolan, A. C., Murphy, M. T., Redmond, L. J. \& Duffield, D. (2009). Maternal characteristics and the production and recruitment of sons in the eastern kingbird (Tyrannus tyrannus). Behavioral Ecology and Sociobiology 63, 1527-1537.

*Donadio, E., Buskirk, S. W. \& Novaro, A. J. (2012). Juvenile and adult mortality patterns in a vicuna (Vicugna vicugna) population. Fournal of Mammalogy 93, 1536-1544.

*Dybala, K. E., Gardali, T. \& Eadie, J. M. (2013). Dependent vs. independent juvenile survival: contrasting drivers of variation and the buffering effect of parental care. Ecology 94, 1584-1593.

Egger, M., Davey Smith, G., Schneider, M. \& Minder, G. (1997). Bias in meta-analysis detected by a simple, graphical test. British Medical Fournal 315, 629-634.

*Ellis, S. L., Bowen, W. D., Boness, D. J. \& Iverson, S. J. (2000). Maternal effects on offspring mass and stage of development at birth in the harbor seal, Phoca vitulina. Journal of Mammalogy 81, 1143-1156.

*Eloranta, E. \& Nieminen, M. (1986). Calving of the experimental reindeer herd in Kaamanen during 1970-1985. Rangifer 1, 115-121.

*Ericsson, G., Wallin, K., Ball, J. P. \& Broberg, M. (2001). Age-related reproductive effort and senescence in free-ranging moose, Alces alces. Ecology 82, $1613-1620$.

*Fairbanks, W. S. (1993). Birthdate, birthweight, and survival in pronghorn fawns. Journal of Mammalogy 74, 129-135.

*Fedak, M., Arnbom, T. \& Boyd, I. (1996). The relation between the size of southern elephant seal mothers, the growth of their pups, and the use of maternal energy, fat, and protein during lactation. Physiological Zoology 69, 887-911.

*Feder, C., Martin, J. G. A., Festa-Bianchet, M., Bérubé, C. \& Jorgenson, J. (2008). Never too late? Consequences of late birthdate for mass and survival of bighorn lambs. Oecologia 156, 773-781.

*Festa-Bianchet, M. \& Jorgenson, J. T. (1998). Selfish mothers: reproductive expenditure and resource availability in bighorn ewes. Behavioral Ecology 9, 144-150.

*Festa-Bianchet, M., Jorgenson, J. T., Bérubé, C. H., Portier, C. \& Wishart, W. D. (1997). Body mass and survival of bighorn sheep. Canadian fournal of Zoology $75,1372-1379$ 
*Forchhammer, M. C., Clutton-Brock, T. H., Lindström, J. \& Albon, S. D. (2001). Climate and population density induce long-term cohort variation in a northern ungulate. Fournal of Animal Ecology 70, 721-729.

Forstmeier, W., Nakagawa, S., Griffith, S. C. \& Kempenaers, B. (2014). Female extra-pair mating: adaptation or genetic constraint? Trends in Ecology \& Evolution 29, $456-464$

*Foster, W. K. \& Taggart, D. A. (2008). Gender and parental influences on the growth of a sexually dimorphic carnivorous marsupial. Fournal of Zoology 275, $221-228$

GADGIL, M. \& Bossert, W. H. (1970). Life historical consequences of natural selection. The American Naturalist 104, 1-24.

Gaillard, J. M., Festa-Bianchet, M., Yoccoz, N., Loison, A. \& Toigo, C. (2000). Temporal variation in fitness components and population dynamics of large herbivores. Annual Review of Ecology, Evolution, and Systematics 31, 367-393.

Garamszegi, L., Eens, M., Hurtrezbousses, S. \& Moller, A. (2005). Testosterone, testes size, and mating success in birds: a comparative study. Hormones and Behavior 47, 389-409.

*Garnett, M. C. (1981). Body size, its heritability and influence on juvenile survival among great tits, Parus major. Ibis 123, 31-41.

Garratt, M., Brooks, R. C., Lemaître, J. F. \& Gaillard, J. M. (2014). Female promiscuity and maternally dependent offspring growth rates in mammals. Evolution 68, $1207-1215$.

Garratt, M., Lemaître, J. F., Douhard, M., Bonenfant, C., Capron, G., Warnant, C., Klein, F., Brooks, R. C. \& Gaillard, J. M. (2015). High juvenile mortality is associated with sex-specific adult survival and lifespan in wild roe deer. Current Biology 25, 759-763.

*Gaston, A. J. (1997). Mass and date at departure affect the survival of ancient murrelet Synthliboramphus antiquus chicks after leaving the colony. Ibis 139, 673-678.

Gelmann, A. \& Rubin, D. B. (1992). Inference from iterative simulation using multiple sequences. Statistical Science 7, 457-511.

Gilroy, J. J., Virzi, T., Boulton, R. L. \& Lockwood, J. L. (2012). A new approach to the 'apparent survival' problem: estimating true survival rates from mark-recapture studies. Ecology 93, 1509-1516.

*Gonzalez, O., Zedrosser, A., Pelletier, F., Swenson, J. E. \& Festa-Bianchet, M. (2012). Litter reductions reveal a trade-off between offspring size and number in brown bears. Behavioral Ecology and Sociobiology 66, 1025-1032.

Gosler, A. G., Greenwood, J. J. D. \& Perrins, C. (1995). Predation risk and the cost of being fat. Nature $377,621-623$.

*Green, D. J. \& Cockburn, A. (2001). Post-fledging care, philopatry and recruitment in brown thornbills. Journal of Animal Ecology 70, 505-514.

*Greño, J. L., Belda, E. J. \& BARba, E. (2008). Influence of temperatures during the nestling period on post-fledging survival of great tit Parus major in a Mediterranean habitat. Fournal of Avian Biology 39, 41-49.

*Griffin, K. A., Hebblewhite, M., Robinson, H. S., Zager, P., Barber-Meyer, S. M., Christianson, D., Creel, S., Harris, N. C., Hurley, M. A., Jackson, D. H., Johnson, B. K., Myers, W. L., Raithel, J. D., Schlegel, M., Smith, B. L., White, C. \& White, P. J. (2011). Neonatal mortality of elk driven by climate, predator phenology and predator community composition. Fournal of Animal Ecology 80, $1246-1257$

*Gustafson, L. L., Franklin, W. L., Johnson, W. E., Behl, M. J., Sarno, R. J., Hunter, R. L. \& Young, K. M. (1998). Predicting early mortality of newborn guanacos by birth mass and hematological parameters: a provisional model. Fournal of Wildlife Management 62, 24-35.

HAdFiELD, J. D. (2010). MCMC methods for multi-response generalized linear mixed models: the MCMCglmm R package. Fournal of Statistical Software 33, 1-22.

HAdFiELd, J. D. \& NAKAgAwA, S. (2010). General quantitative genetic methods for comparative biology: phylogenies, taxonomies and multi-trait models for continuous and categorical characters. Fournal of Evolutionary Biology 23, 494-508.

*Hall, A. J., McConnell, B. J. \& Barker, R. (2001). Factors affecting first-year survival in grey seals and their implications for life history strategy. Fournal of Animal Ecology 70, 138-149.

*Hall, A. J., McConnell, B. J. \& Barker, R. J. (2002). The effect of total immunoglobulin levels, mass and condition on the first-year survival of Grey Seal pups. Functional Ecology 16, 462-474.

*Hamel, S., Craine, J. M. \& Towne, E. G. (2012a). Maternal allocation in bison: co-occurrence of senescence, cost of reproduction, and individual quality. Ecological Applications 22, 1628-1639.

Hamel, S., Yoccoz, N. G. \& Gaillard, J. M. (2012b). Statistical evaluation of parameters estimating autocorrelation and individual heterogeneity in longitudinal studies. Methods in Ecology and Evolution 3, 731-742.

Hamel, S., Gaillard, J. M., Festa-Bianchet, M. \& Côté, S. D. (2009). Individual quality, early-life conditions, and reproductive success in contrasted populations of large herbivores. Ecology 90, 1981-1995.

Hamel, S., Gaillard, J. M., Yoccoz, N. G., Loison, A., Bonenfant, G. \& Descamps, S. (2010). Fitness costs of reproduction depend on life speed: empirical evidence from mammalian populations. Ecology Letters 13, 915-935.

*Harris, M. P. \& Rothery, P. (1985). The post-fledging survival of young Puffins Fratercula arctica in relation to hatching date and growth. Ibis 127, 243-250.
Harvey, P. H. \& Pagel, M. D. (1991). The Comparative Method in Evolutionary Biology. Oxford University Press, Oxford.

*Hedgren, S. (1981). Effects of fledging weight and time of fledging on survival of guillemot Uria aalge chicks. Ormis Scandinavica 12, 51-54.

*Helle, S., Laaksonen, T. \& Huitu, O. (2013). Sex-specific offspring growth according to maternal testosterone, corticosterone, and glucose levels. Behavioral Ecology 24, 205-212.

*Hewison, A. J. M., Gaillard, J. M., Kjellander, P., Toigo, C., Liberg, O. \& Delorme, D. (2005). Big mothers invest more in daughters - reversed sex allocation in a weakly polygynous mammal. Ecology Letters 8, 430-437.

Higgins, J. P. T., Thompson, S. G., Deeks, J.J. \& Altman, D. G. (2003). Measuring inconsistency in meta-analyses. British Medical fournal 327, 557-560.

*Hodge, S. J., Bell, M. B. V., Mwanguhya, F., Kyabulima, S., Waldick, R. C. \& Russell, A. F. (2009). Maternal weight, offspring competitive ability, and the evolution of communal breeding. Behavioral Ecology 20, 729-735.

*Hoffman, J. I., Forcada, J. \& Amos, W. (2006). No relationship between microsatellite variation and neonatal fitness in Antarctic fur seals, Arctocephalus gazella. Molecular Ecology 15, 1995-2005.

*Holand, Ø., Mysterud, A., Roed, K. H., Coulson, T., Gjostein, H., Weladji, R. B. \& Nieminen, M. (2006). Adaptive adjustment of offspring sex ratio and maternal reproductive effort in an iteroparous mammal. Proceedings of the Royal Society B: Biological Sciences 273, 293-299.

*Holand, Ø., Weladji, R. B., Gjøstein, H., Kumpula, J., Smith, M. E., Nieminen, M. \& RøED, K. H. (2004). Reproductive effort in relation to maternal social rank in reindeer (Rangifer tarandus). Behavioral Ecology and Sociobiology 57, 69-76.

*Horswill, C., Matthiopoulos, J., Green, J. A., Meredith, M. P., Forcada, J., Peat, H., Preston, M., Trathan, P. N. \& Ratcliffe, N. (2014). Survival in macaroni penguins and the relative importance of different drivers: individual traits, predation pressure and environmental variability. Fournal of Animal Ecology 83 $1057-1067$

Hurlbert, S. H. (1984). Pseudoreplication and the design of ecological field experiments. Ecological Monographs 54, 187.

*Hurley, M. A., Unsworth, J. W., Zager, P., Hebblewhite, M., Garton, E. O., Montgomery, D. M., Skalski, J. R. \& Maycock, C. L. (2011). Demographic response of mule deer to experimental reduction of coyotes and mountain lions in southeastern Idaho. Wildlife Monographs 178, 1-33.

*Husby, M. \& Slagsvold, T. (1992). Post-fledging behavior and survival in male and female behaviour Magpies Pica pica. Ornis Scandinavica 23, 483-490.

*Iverson, S. J., Bowen, W. D., Boness, D. J. \& Oftedal, O. T. (1993). The effects of maternal size and milk energy output on pup growth in grey seals (Halichoerus gyppus). Physiological Zoology 66, 61-88.

Jašarević, E., Bailey, D. H., Crossland, J. P., Dawson, W. D., Szalai, G., Ellersieck, M. R., Rosenfeld, C. S. \& Geary, D. C. (2013). Evolution of monogamy, paternal investment, and female life history in Peromyscus. Fournal of Comparative Psychology 127, 91-102.

*Jenkins, K. J. \& Barten, N. L. (2005). Demography and decline of the Mentasta caribou herd in Alaska. Canadian Fournal of Zoology 83, 1174-1188.

Jetz, W., Thomas, G. H. H., Joy, J. B. B., Hartmann, K. \& Mooers, A. O. O. (2012). The global diversity of birds in space and time. Nature 491, 1-5.

*Jones, O. R., Crawley, M. J., Pilkington, J. G. \& Pemberton, J. M. (2005). Predictors of early survival in Soay sheep: cohort-, maternal- and individual-level variation. Proceedings of the Royal Society B: Biological Sciences 272, 2619-2625.

*Kasparian, K., Geissler, E. \& Trillmich, F. (2005). Optimal offspring size in a small mammal: an exception to the tradeoff invariant life-history rule. Oikos 111, $271-278$.

*Keech, M. A., Bowyer, R. T., Hoef, J. M. V., Boertje, R. D., Dale, B. W. \& STEPhenson, T. R. (2000). Life-history consequences of maternal condition in Alaskan Moose. Journal of Wildlife Management 64, 450.

*Keech, M. A., Lindberg, M. S., Boertje, R. D., Valkenburg, P., Taras, B. D., Boudreau, T. A. \& Beckmen, K. B. (2011). Effects of predator treatments, individual traits, and environment on moose survival in Alaska. Journal of Wildlife Management 75, 1361-1380.

*Kershner, E. L., Walk, J. W. \& Warner, R. E. (2004). Postfledging movements and survival of juvenile eastern meadowlarks (Sturnella magna) in Illinois. The Auk 121, $1146-1154$

*Kersten, M. \& Brenninkmeijer, A. (1995). Growth, fledging success and post-fledging survival of juvenile oystercatchers Haematopus ostralegus. Ibis 137, $396-404$.

*Kojola, I. (1993). Early maternal investment and growth in reindeer. Canadian fournal of Zoology 71, 753-758.

*Koskela, E., Huitu, O., Koivula, M., Korpimaki, E. \& Mappes, T. (2004). Sex-biased maternal investment in voles: importance of environmental conditions. Proceedings of the Royal Society B: Biological Sciences 271, 1385-1391.

*Krackow, S. (1997). Maternal investment, sex-differential prospects, and the sex ratio in wild house mice. Behavioral Ecology and Sociobiology 41, 435-443.

*Kraus, C., Mueller, B., Meise, K., Piedrahita, P., Pörschmann, U. \& Trillmich, F. (2013). Mama's boy: sex differences in juvenile survival in a highly dimorphic large mammal, the Galapagos sea lion. Oecologia 171, 893-903. 
*Krebs, E. A. (1999). Last but not least: nestling growth and survival in asynchronously hatching crimson rosellas. Foumal of Animal Ecology 68, 266-281.

KrIst, M. (2011). Egg size and offspring quality: a meta-analysis in birds. Biological Reviewes 86, 692-716

Labocha, M. K. \& Hayes, J. P. (2012). Morphometric indices of body condition in birds: a review. Fournal of Ornithology 153, 1-22.

*Landete-Castillejos, T., García, A., Gómez, J. Á., Molina, A. \& Gallego, L. (2003). Subspecies and body size allometry affect milk production and composition, and calf growth in red deer: comparison of Cervus elaphus hispanicus and Cervus elaphus scoticus. Physiological and Biochemical Zoology 76, 594-602.

*Landete-Castillejos, T., García, A., López-Serrano, F. R. \& Gallego, L. (2005). Maternal quality and differences in milk production and composition for male and female Iberian red deer calves (Cervus elaphus hispanicus). Behavioral Ecology) and Sociobiology 57, 267-274.

*Larivée, M. L., Boutin, S., Speakman, J. R., McAdam, A. G. \& Humphries, M. M. (2010). Associations between over-winter survival and resting metabolic rate in juvenile North American red squirrels. Functional Ecology 24, 597-607.

Lebreton, J. D., Burnham, K. P., Clobert, J. \& Anderson, D. R. (1992). Modeling survival and testing biological hypotheses using marked animals: a unified approach with case studies. Ecological Monographs 62, 67.

Lebreton, J. D., Pradel, R. \& Clobert, J. (1993). The statistical analysis of survival in animal populations. Trends in Ecology \& Evolution 8, 91-95.

Lee, P. C., Majluf, P. \& Gordon, I. J. (1991). Growth, weaning and maternal investment from a comparative perspective. Fournal of Zoology 225, 99-114.

*LegGE, S. (2002). Siblicide, starvation and nestling growth in the laughing kookaburra. Journal of Avian Biology 33, 159-166.

Lemaître, J. F., Gaillard, J. M., Lackey, L. B., Clauss, M. \& Müller, D. W. H. (2013). Comparing free-ranging and captive populations reveals intra-specific variation in aging rates in large herbivores. Experimental Gerontology 48, 162-167.

Liberati, A., Altman, D. G., Tetzlaff, J., Mulrow, C., Gotzsche, P. C., Ioannidis, J. P. A., Clarke, M., Devereaux, P. J., Kleijnen, J. \& Moher, D. (2009). The PRISMA statement for reporting systematic reviews and meta-analyses of studies that evaluate healthcare interventions: explanation and elaboration. British Medical fournal 339, b2700.

Lim, J. N., Senior, A. M. \& Nakagawa, S. (2014). Heterogeneity in individual quality and reproductive trade-offs within species. Evolution $\mathbf{6 8 , 2 3 0 6 - 2 3 1 8 .}$

*Lindén, M., Gustafsson, L. \& Part, T. (1992). Selection on fledging mass in the collared flycatcher and the great tit. Ecology 73, 336.

LindSTRöm, J. (1999). Early development and fitness in birds and mammals. Trends in Ecology and Evolution 14, 343-348.

Linnell, J. D. C., Aanes, R. \& Andersen, R. (1995). Who killed Bambi? The role of predation in the neonatal mortality of temperate ungulates. Wildlife Biology $\mathbf{1}$, 209-223.

Lipsey, M. W. \& Wilson, D. B. (2001). Practical Meta-analysis. Sage Publications, Thousand Oaks.

Littleton, J. (2005). Fifty years of Chimpanzee demography at Taronga Park Zoo. American Fournal of Primatology 67, 281-298.

*Lloyd, G. S. (1979). Factors affecting breeding of razorbills Alca torda on Skokholm. Ibis 121, 165-176.

*Lloyd, P., Andrew, T. W., Du Plessis, M. A. \& Martin, T. E. (2009). Females increase reproductive investment in response to helper-mediated improvements in allo-feeding, nest survival, nestling provisioning and post-fledging survival in the Karoo scrub-robin Cercotrichas coryphaeus. Fournal of Avian Biology 40, 400-411.

*Lobato, E., Moreno, J., Merino, S., Sanz, J. J. \& Arriero, E. (2005). Haematological variables are good predictors of recruitment in nestling pied flycatchers (Ficedula hypoleuca). Ecoscience 12, 27-34.

*Loison, A., Langvatn, R. \& Solberg, E. J. (1999). Body mass and winter mortality in red deer calves: disentangling sex and climate effects. Ecography 22, 20-30.

*Lomas, L. A. \& Bender, L. C. (2007). Survival and cause-specific mortality of neonatal mule deer fawns, north-central New Mexico. Journal of Wildlife Management 71, 884-894.

*LunN, N. J. \& Boyd, I. L. (1993). Effects of maternal age and condition on parturition and the perinatal period of Antarctic fur seals. Fournal of Zoology 229, 55-67.

*Lusk, J. J., Guthery, F. S., Cox, S. A., Demaso, S. J. \& Peoples, A. D. (2005). Survival and growth of northern bobwhite chicks in western Oklahoma. The American Midland Naturalist 153, 389-395.

* Mabille, G. \& Berteaux, D. (2014). Hide or die: use of cover decreases predation risk in juvenile North American porcupines. Fournal of Mammalogy 95, 992-1003.

*Mackas, R. H., Green, D. J., Whitehorne, I. B. J., Fairhurst, E. N., Middleton, H. A. \& Morrissey, C. A. (2010). Altitudinal migration in American Dippers (Cinclus mexicanus): do migrants produce higher quality offspring? Canadian Journal of Zoology 88, 369-377.

* Magrath, R. D. (1991). Nestling weight and juvenile survival in the blackbird, Turdus merula. Fournal of Animal Ecology 60, 335-351.

Mandal, A., Prasad, H., Kumar, A., Roy, R. \& Sharma, N. (2007). Factors associated with lamb mortalities in Muzaffarnagari sheep. Small Ruminant Research 71, 273-279.
*Maness, T. J. \& Anderson, D. J. (2013). Predictors of juvenile survival in birds. Ornithological Monographs 78, 1-55.

*Maniscalco, J. M. (2014). The effects of birth weight and maternal care on survival of juvenile Steller sea lions (Eumetopias jubatus). PLoS ONE 9, e96328.

*Martín, C. A., Alonso, J. C., Alonso, J. A., Palacín, C., Magaña, M. \& Martín, B. (2007). Sex-biased juvenile survival in a bird with extreme size dimorphism, the great bustard Otis tarda. Fournal of Avian Biology 38, 335-346.

*McCann, T. S., Fedak, M. A. \& Harwood, J. (1989). Parental investment in southern elephant seals, Mirounga leonina. Behavioral Ecology and Sociobiology 25, 81-87.

*McClung, M. R., Seddon, P. J., Massaro, M. \& Setiawan, A. N. (2004). Nature-based tourism impacts on yellow-eyed penguins Megadyptes antipodes: does unregulated visitor access affect fledging weight and juvenile survival? Biological Conservation 119, 279-285.

*McDonald, B. I., Goebel, M. E., Crocker, D. E. \& Costa, D. P. (2012). Dynamic influence of maternal and pup traits on maternal care during lactation in an income breeder, the Antarctic fur seal. Physiological and Biochemical Zoology 85, 243-254.

*McMahon, C. R., Burton, H. R. \& Bester, M. N. (2000). Weaning mass and the future survival of juvenile southern elephant seals, Mirounga leonina, at Macquarie Island. Antarctic Science 12, 149-153.

McMahon, C. R., New, L. F., Fairley, E. J., Hindell, M. A. \& Burton, H. R. (2015). The effects of body size and climate on post-weaning survival of elephant seals at Heard Island. Fournal of Zoology 297, 301-308.

*Medeiros, M. C. \& Freed, L. A. (2009). A fledgling-mass threshold greatly affects juvenile survival in the Hawaii akepa (Loxops coccineus coccineus). The Auk 126, 319-325.

Menard, S. (2011). Standards for standardized logistic regression coefficients. Social Forces 89, 1409-1428.

*Michel, E. S., Demarais, S., Strickland, B. K. \& Belant, J. L. (2015). Contrasting the effects of maternal and behavioral characteristics on fawn birth mass in white-tailed deer. PLOS ONE 10, e0136034.

Michener, G. R. (1989). Reproductive effort during gestation and lactation by Richardson's ground squirrels. Oecologia 78, 77-86.

*Monclús, R., Pang, B. \& Blumstein, D. T. (2014). Yellow-bellied marmots do not compensate for a late start: the role of maternal allocation in shaping life-history trajectories. Evolutionary Ecology 28, 721-733.

*Monrós, J. S., Belda, E. J. \& Barba, E. (2002). Post-fledging survival of individual great tits: the effect of hatching date and fledging mass. Oikos 99, 481-488.

Monteith, K. L., Bleich, V. C., Stephenson, T. R., Pierce, B. M., Conner, M. M., KIE, J. G. \& Bowyer, R. T. (2014). Life-history characteristics of mule deer: effects of nutrition in a variable environment. Wildlife Monographs 186, 1-62.

*Monticelli, D. \& Ramos, J. A. (2012). Laying date, body mass and tick infestation of nestling tropical Roseate Terns Sterna dougallii predict fledging success, first-year survival and age at first return to the natal colony. Ibis 154, 825-837.

*Moore, G. H., Littlejohn, R. P. \& Cowie, G. M. (1988). Factors affecting live weight gain in red deer calves from birth to weaning. New Zealand Journal of Agricultural Research 31, 279-283.

*Morrison, K. W., Hipfner, J. M., Gjerdrum, C. \& Green, D. J. (2009). Wing length and mass at fledging predict local juvenile survival and age at first return in tufted puffins. The Condor 111, 433-441.

*Moyes, K., Nussey, D. H., Clements, M. N., Guinness, F. E., Morris, A., Morris, S., Pemberton, J. M., Kruuk, L. E. B. \& Clutton-Brock, T. H. (2011). Advancing breeding phenology in response to environmental change in a wild red deer population. Global Change Biology 17, 2455-2469.

*Mukasa-Mugerwa, E., Said, A. N., Lahlou-Kassi, A., Sherington, J. \& Mutiga, E. R. (1994). Birth weight as a risk factor for perinatal lamb mortality, and the effects of stage of pregnant ewe supplementation and gestation weight gain in Ethiopian Menz sheep. Preventive Veterinary Medicine 19, 45-56.

*Mumme, R. L., Bowman, R., Pruett, M. S. \& Fitzpatrick, J. W. (2015). Natal territory size, group size, and body mass affect lifetime fitness in the cooperatively breeding Florida Scrub-Jay. The Auk 132, 634-646.

*Myers, P. \& Master, L. L. (1983). Reproduction by Peromyscus maniculatus: size and compromise. Fournal of Mammalogy 64, 1-18.

*Mysterud, A., Røed, K. H., Holand, Ø., Yoccoz, N. G. \& Nieminen, M. (2009). Age-related gestation length adjustment in a large iteroparous mammal at northern latitude. Fournal of Animal Ecology 78, 1002-1006.

*Naef-Daenzer, B., Widmer, F. \& Nuber, M. (2001). Differential post fledging survival of great and coal tits in relation to their condition and fledging date. Fournal of Animal Ecology 70, 730-738.

NAkagawa, S. \& Poulin, R. (2012). Meta-analytic insights into evolutionary ecology: an introduction and synthesis. Evolutionary Ecology 26, 1085-1099.

Nakagawa, S. \& Santos, E. S. A. (2012). Methodological issues and advances in biological meta-analysis. Evolutionary Ecology 26, 1253-1274.

*Nespolo, R. F. \& Bacigalupe, L. D. (2009). Viability selection on early body mass and the effect of female body size on fecundity: a study on the leaf-eared mouse Phyllotis darwini. Ecological Research 24, 997-1002.

Newbrey, J. L. \& Reed, W. L. (2009). Growth of yellow-headed blackbird Xanthocephalus xanthocephalus nestlings in relation to maternal body condition, egg mass, and yolk carotenoids concentrations. Fournal of Avian Biology 40, 419-429. 
Norberg, U. M. (1995). How a long tail and changes in mass and wing shape affect the cost for flight in animals. Functional Ecology 9, 48

*Noyce, V., Coy, L. \& Garshelis, L. (2002). Bone prominence and skin-fold thickness as predictors of body fat and reproduction in American black bears. Ursus 13, 275-284.

*Nur, N. (1984). The consequences of brood size for breeding blue tits II. Nestling weight, offspring survival and optimal brood size. Journal of Animal Ecology 53, $497-517$.

*Olsson, O. (1997). Effects of food availability on fledging condition and post-fledging survival in king penguin chicks. Polar Biology 18, 161-165.

*Owen, B. Y. M. \& Black, J. M. (1989). Factors affecting the survival of barnacle geese on migration from the breeding grounds. Fournal of Animal Ecology 58, 603-617.

Ozgul, A., Childs, D. Z., Oli, M. K., Armitage, K. B., Blumstein, D. T., Olson, L. E., Tuljapurkar, S. \& Coulson, T. (2010). Coupled dynamics of body mass and population growth in response to environmental change. Nature 466 , $482-485$

Parker, T. H. (2002). Maternal condition, reproductive investment, and offspring sex ratio in captive red junglefowl (Gallus gallus). The Auk 119, 840.

Parker, K. L., Barboza, P. S. \& Gillingham, M. P. (2009). Nutrition integrates environmental responses of ungulates. Functional Ecology 23, 57-69.

*Patterson, I. J., Dunnet, G. M. \& Goodbody, S. R. (1988). Body weight and juvenile mortality. Fournal of Animal Ecology 57, 1041-1052.

*Perrins, G. M., Harris, M. P. \& Britton, C. K. (1973). Survival of Manx shearwaters. Ibis 115, 535-548.

*Plard, F., Gaillard, J. M., Coulson, T., Hewison, M. A. J., Douhard, M., Klein, F., Delorme, D., Warnant, C. \& Bonenfant, C. (2015). The influence of birth date via body mass on individual fitness in a long-lived mammal. Ecology $\mathbf{9 6}$, $1516-1528$.

*Pomeroy, P. P., Fedak, M. A., Rothery, P. \& Anderson, S. (1999). Consequences of maternal size for reproductive expenditure and pupping success of grey seals at North Rona, Scotland. Fournal of Animal Ecology 68, 235-253.

*Ponz Miranda, A., Gil-Delgado Alberti, J. A. \& López Iborra, M. (2007). Survival rates of young magpies Pica pica in a mountain population of eastern Spain. Acta Ornithologica 42, 63-68.

*Postma, M., Bester, M. N. \& de Bruyn, P. J. N. (2013). Age-related reproductive variation in a wild marine mammal population. Polar Biology 36, 719-729.

*Potti, J., Dávila, J. A., Tella, J. L., Frís, O.ć. \& Villar, S. (2002). Gender and viability selection on morphology in fledgling pied flycatchers. Molecular Ecology 11, $1317-1326$.

*Proffitt, K. M., Garrott, R. A. \& Rotella, J. J. (2008). Long-term evaluation of body mass at weaning and postweaning survival rates of Weddell seals in Erebus Bay, Antarctica. Marine Mammal Science 24, 677-689.

*Raberg, L., Stjernman, M. \& Nilsson, J. (2005). Sex and environmental sensitivity in blue tit nestlings. Oecologia 145, 496-503.

R Core Development Team (2015). R: A Language and Environment for Statistical Computing. R Foundation for Statistical Computing, Vienna. Available at http://www.Rproject.org/.

*Ramsay, M. A. \& STIRLING, I. (1988). Reproductive biology and ecology of female polar bears (Ursus maritimus). Fournal of the Zoology 214, 601-634.

*Reading, R. P., Kenny, D., Amgalanbatar, S., Denicola, A. \& Wingard, G. (2009). Argali lamb (Ovis ammon) morphometric measurements and survivorship in Mongolia. Mammalia 73, 98-104

*Riesch, R., Martin, R. A., Lerp, H., Plath, M. \& Wronski, T. (2013). Size and sex matter: reproductive biology and determinants of offspring survival in Gazella marica. Biological fournal of the Linnean Society 110, 116-127.

*Ringsby, T. H., Saether, B. E. \& Solberg, E. J. (1998). Factors affecting juvenile survival in house sparrow Passer domesticus. Foumal of Avian Biology 29, 241.

*Rioux-Paquette, E., Festa-Bianchet, M. \& Coltman, D. W. (2011). Sex-differential effects of inbreeding on overwinter survival, birth date and mass of bighorn lambs. Fournal of Evolutionary Biology 24, 121-131.

*Robbins, C. T., Ben-David, M., Fortin, J. K. \& Nelson, O. L. (2012). Maternal condition determines birth date and growth of newborn bear cubs. Fournal of Mammalogy 93, 540-546.

*Rödel, H. G., Bora, A., Kaetzke, P., Khaschei, M., Hutzelmeyer, H. \& Von Holst, D. (2004). Over-winter survival in subadult European rabbits: weather effects, density dependence, and the impact of individual characteristics. Oecologia 140, 566-576.

*Rödel, H. G., Starkloff, A., Seltmann, M. W., Prager, G. \& von Holst, D. (2009). Causes and predictors of nest mortality in a European rabbit population. Mammalian Biology 74, 198-209.

Rogers, C. M. (2015). Testing optimal body mass theory: evidence for cost of fat in wintering birds. Ecosphere 6, 1-12.

*Rognmo, A., Markussen, K. A., Jacobsen, E., Grav, H. J. \& Blix, A. S. (1983). Effects of improved nutrition in pregnant reindeer on milk quality, calf birth weight, growth, and mortality. Rangifer 3, 10-18.

Rosenthal, R. (1979). The file drawer problem and tolerance for null results. Psychological Bulletin 86, 638-641.
*Rotella, J. J., Clark, R. G. \& Afron, A. D. (2003). Survival of female lesser scaup: effects of body size, age, and reproductive effort. The Condor 105, 336-347.

*Russell, A. F., Brotherton, P. N. M., McIlrath, G. M., Sharpe, L. L. \& Clutton-Brock, T. H. (2003). Breeding success in cooperative meerkats: effects of helper number and maternal state. Behavioral Ecology 14, 486-492.

Rutkowska, J., Dubiec, A. \& Nakagawa, S. (2014). All eggs are made equal: meta-analysis of egg sexual size dimorphism in birds. Fournal of Evolutionary Biology 27, 153-160.

*Sagar, P. M. \& Horning, D. S. (1998). Mass-related survival of fledgling Sooty shearwaters Puffinus griseus at The Snares, New Zealand. Ibis 140, 329-331.

*Sams, M. G., Lochmiller, R. L., Qualls, C. W. J., Leslie, D. M. J. \& Payton, M. E. (1996). Physiological correlates of neonatal mortality in an overpopulated herd of white tailed deer. Fournal of Mammalogy 77, 179-190.

Santos, E. S. A. \& NAKagawa, S. (2012). The costs of parental care: a meta-analysis of the trade-off between parental effort and survival in birds. Fournal of Evolutionary Biology 25, 1911-1917.

*Schauroth, C. \& Becker, P. H. (2008). Post-fledging body mass increase in common terns Sterna hirundo: influence of age, sex and year. Ibis 150, 50-58.

Schindler, S., Gaillard, J. M., Grüning, A., Neuhaus, P., Traill, L. W., Tuljapurkar, S. \& Coulson, T. (2015). Sex-specific demography and generalization of the Trivers-Willard theory. Nature 526, 249-252.

*Schmutz, J. A. (1993). Survival and pre-fledging body mass in juvenile emperor geese. The Condor 95, 222-225.

Schulte-Hostedde, A. I., Zinner, B., Millar, J. S. \& Graham, J. H. (2005). Restitution of mass-size residuals: validating body condition indices. Ecology $\mathbf{8 6}$ $155-163$.

*Serra, L., Pirrello, S., Caprioli, M., Griggio, M., Andreotti, A., Romano, A., Pilastro, A., Saino, N., Sacchi, R., Galeotti, P., Fasola, M., Spina, F. \& Rubolini, D. (2012). Seasonal decline of offspring quality in the European starling Sturnus vulgaris: an immune challenge experiment. Behavioral Ecology and Sociobiology 66, 697-709.

*Setchell, J. M., Lee, P. C., Wickings, E. J. \& Dixson, A. F. (2001). Growth and ontogeny of sexual size dimorphism in the mandrill (Mandrillus sphinx). American Fournal of Physical Anthropology 115, 349-360.

*Shaughnessy, P. W., Digiacomo, R. F., Martin, D. P. \& Valerio, D. A. (1978). Prematurity and perinatal mortality in the rhesus (Macaca mulatta): relationship to birth weight and gestational age. Neonatology 34, 129-145.

*Shibata, F. \& Kawamichi, T. (2009). Female-biased sex allocation of offspring by an Apodemus mouse in an unstable environment. Behavioral Ecology and Sociobiology 63, $1307-1317$

*Skibiel, A. L., Dobson, F. S. \& Murie, J. O. (2009). Maternal influences on reproduction in two populations of Columbian ground squirrels. Ecological Monographs 79, 325-341.

SkogLand, T. (1984). The effects of food and maternal conditions in fetal growth and size in wild reindeer. Rangifer 4, 39.

Smith, G. C. \& Fretwell, S. D. (1974). The optimal balance between size and number of offspring. The American Naturalist 108, 499-506.

*Smith, H., Kallander, H. \& Nilsson, J.-A. (1989). The trade-off between offspring number and quality in the great tit Parus major. Fournal of Animal Ecology 58, 383-401.

*SNow, D. W. (1958). The breeding of the Blackbird Turdus merula at Oxford. Ibis 100 $1-30$

*Soler, M., Palomino, J. \& Martinez, J. G. (1994). Activity, survival, independance and migration of fledging great spotted cuckoos. The Condor 96, 802-805.

*Souchay, G., Gauthier, G. \& Pradel, R. (2013). Temporal variation of juvenile survival in a long-lived species: the role of parasites and body condition. Oecologia $173,151-160$.

Stearns, S. C. (1992). The Evolution of Life Histories. Oxford University Press, Oxford.

*Steinheim, G., Mysterud, A., Holand, Ø., Bakken, M. \& Adnoy, T. (2002). The effect of initial weight of the ewe on later reproductive effort in domestic sheep (Ovis aries). Journal of Zoology 258, 515-520.

*Stienen, E. W. M. \& Brenninkmeijer, A. (2002). Variation in growth in sandwich tern chicks Sterna sandvicensis and the consequences for pre- and post-fledging mortality. Ibis 144, 567-576.

Stoleson, S. H. \& Beissinger, S. R. (1997). Hatching asynchrony, brood reduction, and food limitation in a neotropical parrot. Ecological Monographs 67, 131-154.

*Stopher, K. V., Bento, A. I., Clutton-Brock, T. H., Pemberton, J. M. \& KruUK, L. E. B. (2014). Multiple pathways mediate effects of climate change on maternal reproductive traits in a red deer population. Ecology 95, 3124-3138.

*Suedkamp Wells, K. M., Ryan, M. R., Millspaugh, J. J., Thompson, F. R. \& Hubbard, M. W. (2007). Survival of postfledging grassland birds in Missouri. The Condor 109, 781.

*Sullivan, K. A. (1989). Predation and starvation: age-specific mortality in juvenile juncos (Funco phaenotus). Fournal of Animal Ecology 58, 275-286.

*Taillon, J., Brodeur, V., Festa-Bianchet, M. \& Côté, S. D. (2012). Is mother condition related to offspring condition in migratory caribou (Rangifer tarandus) at calving and weaning? Canadian fournal of Zoology 90, 393-402. 
Tavecchia, G., Coulson, T., Morgan, B. J. T., Pemberton, J. M., Pilkington, J. C., Gulland, F. M. D. \& Clutton-Brock, T. H. (2005). Predictors of reproductive cost in female Soay sheep. Journal of Animal Ecology 74, 201-213.

Tidière, M., Gaillard, J. M., Berger, V., Müller, D. W. H., Bingaman Lackey, L., Gimenez, O., Clauss, M. \& Lemaître, J. F. (2016). Comparative analyses of longevity and senescence reveal variable survival benefits of living in zoos across mammals. Scientific Reports 6, 36361.

*Tobey, J. R., Andrus, C. H., Doyle, L., Thompson, V. D. \& Bercovitch, F. B. (2006). Maternal effort and joey growth in koalas (Phascolarctos cinereus). Fournal of Zoology 268, 423-431

*Todd, L. D., Poulin, R. G., Wellicome, T. I. \& Brigham, R. M. (2003). Post-fledging survival of burrowing owls in Saskatchewan. Journal of Wildlife Management 67, 512-519.

*Traylor, J. J. \& Alisauskas, R. T. (2006). Effects of intrinsic and extrinsic factors on survival of white-winged scoter (Melanitta fusca deglandi) ducklings. The Auk $\mathbf{1 2 3}$ $67-81$.

Trivers, R. L. \& WiLLaRd, D. E. (1973). Natural selection of parental ability to vary the sex ratio of offspring. Science 179, 90-92.

*VAn der Jeugd, H. \& Larsson, K. (1998). Pre-breeding survival of barnacle geese Branta leucopsis in relation to fledgling characteristics. Fournal of Animal Ecology 67, 953-966.

*Van Vuren, D. H., Bray, M. P. \& Heltzel, J. M. (2013). Differential investment in twin offspring by female pronghorns (Antilocapra americana). Fournal of Mammalogy 94, 155-161.

*Verboven, N. \& Visser, M. E. (1998). Seasonal variation in local recruitment of great tits: the importance of being early. Oikos 81, 511-524.

Viechtbauer, W. (2010). Conducting meta-analyses in R with the metafor Package. Journal of Statistical Software 36, 1-48.

*VItz, A. C. \& Rodewald, A. D. (2011). Influence of condition and habitat use on survival of post-fledging songbirds. The Condor 113, 400-411.

*Walling, C. A., Nussey, D. H., Morris, A., Clutton-Brock, T. H., Kruuk, L. E. \& Pemberton, J. M. (2011). Inbreeding depression in red deer calves. BMC Evolutionary Biology 11, 318 .

Warren, J. T., Mysterud, I. \& Lynnebakken, T. (2001). Mortality of lambs in free-ranging domestic sheep (Ovis aries) in northern Norway. Fournal of Zoology 254, $195-202$.

*Wauters, L., Bijnens, L. \& Dhondt, A. A. (1993). Body mass at weaning and juvenile recruitment in the red squirrel. Fournal of Animal Ecology 62, 280-286.

*Weimerskirch, H., Barbraud, C. \& Lys, P. (2000). Sex differences in parental investment and chick growth in wandering albatrosses: fitness consequences. Ecology 81, 309-318.

*Wheatley, K. E., Bradshaw, C. J. A., Davis, L. S., Harcourt, R. G. \& Hindell, M. A. (2006). Influence of maternal mass and condition on energy transfer in Weddell seals. Foumal of Animal Ecology 75, 724-733.

*White, G. C., Garrott, R. A., Bartmann, R. M. \& Carpenter, L. H. (1987). Survival of mule deer in northwest Colorado. Journal of Wildlife Management 51, 852-859.

*White, C. G., Zager, P. \& Gratson, M. W. (2010). Influence of predator harvest, biological factors, and landscape on elk calf survival in Idaho. Fournal of Wildlife Management 74, 355-369.
*Whitten, K., Garner, G., Mauer, F. \& Harris, R. (1992). Productivity and early calf survival in the Porcupine caribou herd. Fournal of Wildlife Management 56, $201-212$.

*Wiens, J. D., Noon, B. R. \& Reynolds, R. T. (2006). Post-fledging survival of northern Goshawks: the importance of prey abundance, weather, and dispersal. Ecological Applications 16, 406-418.

Wiggins, D. A. (1990). Sources of variation in egg mass of tree swallows Tachycineta bicolor. Ornis Scandinavica 21, 157.

Wilder, S. M., Raubenheimer, D. \& Simpson, S. J. (2016). Moving beyond body condition indices as an estimate of fitness in ecological and evolutionary studies. Functional Ecology 30, 108-115.

*Williams, T. D. \& Croxall, J. P. (1991). Chick growth and survival in gentoo penguins (Pygoscelis papua): effect of hatching asynchrony and variation in food supply. Polar Biology 11, 197-202.

*Wilson, A.J., Pilkington, J. G., Pemberton, J. M., Coltman, D. W., Overall, A. D. J., Byrne, K. A. \& KruUk, L. E. B. (2005). Selection on mothers and offspring: whose phenotype is it and does it matter? Evolution 59, 451-463.

Wright, J. \& Cotton, P. A. (1994). Experimentally induced sex differences in parental care: an effect of certainty of paternity? Animal Behaviour 47, 1311-1322.

Yapi, C. V., Boylan, W. J. \& Robinson, R. A. (1990). Factors associated with causes of preweaning lamb mortality. Preventive Veterinary Medicine 10, 145-152.

Ylönen, H., Horne, T. J. \& LuUkKonen, M. (2004). Effect of birth and weaning mass on growth, survival and reproduction in the bank vole. Evolutionary Ecology Research 6, 433-442.

Zeveloff, S. I. \& Boyce, M. S. (1980). Parental investment and mating systems in mammals. Evolution 34, 973-982.

\section{SUPPORTING INFORMATION}

Additional supporting information may be found in the online version of this article.

Appendix S1. Data used for the analysis of the relationship between offspring body mass and offspring survival in mammals.

Appendix S2. Data used for the analysis of the relationship between offspring body mass and offspring survival in birds.

Appendix S3. Data used for the analysis of the relationship between maternal mass and offspring mass in mammals.

Appendix S4. R code for the extraction of the effect size for the relationship between offspring mass and offspring survival.

(Received 19 October 2016; revised 23 February 2017; accepted 3 March 2017; published online 9 April 2017) 